\title{
Hybrid Gaussian-B-spline basis for the electronic continuum: Photoionization of atomic hydrogen
}

\author{
Carlos Marante, ${ }^{1}$ Luca Argenti, ${ }^{1, *}$ and Fernando Martín ${ }^{1,2}$ \\ ${ }^{1}$ Departamento de Química, Módulo 13, Universidad Autónoma de Madrid, 28049 Madrid, Spain, EU \\ ${ }^{2}$ Instituto Madrileño de Estudios Avanzados en Nanociencia (IMDEA-Nanociencia), Cantoblanco, 28049 Madrid, Spain, EU
}

(Received 14 February 2014; revised manuscript received 29 May 2014; published 8 July 2014)

\begin{abstract}
As a first step towards meeting the recent demand for new computational tools capable of reproducing molecular-ionization continua in a wide energy range, we introduce a hybrid Gaussian- $B$-spline basis (GABS) that combines short-range Gaussian functions, compatible with standard quantum-chemistry computational codes, with $B$ splines, a basis appropriate to represent electronic continua. We illustrate the performance of the GABS hybrid basis for the hydrogen atom by solving both the time-independent and the time-dependent Schrödinger equation for a few representative cases. The results are in excellent agreement with those obtained with a purely $B$-spline basis, with analytical results, when available, and with recent above-threshold ionization spectra from the literature. In the latter case, we report fully differential photoelectron distributions which offer further insight into the process of above-threshold ionization at different wavelengths.
\end{abstract}

DOI: 10.1103/PhysRevA.90.012506

PACS number(s): 31.15.-p, 32.80.Fb, 32.80.Rm, 32.70.Cs

\section{INTRODUCTION}

With the advent of subfemtosecond extreme ultraviolet light pulses from high-harmonic generation [1] and femtosecond intense $\mathrm{X}$-ray sources [2], in combination with high-resolution techniques to detect photoelectrons [3] and ions in coincidence [4], as well as to monitor the light transmitted through the sample [5-9], it is now possible to study the ultrafast dynamics of electronic and nuclear motion in atoms and molecules with unprecedented sensitivity and time resolution.

While these advances offer the opportunity to probe increasingly more complex phenomena, however, they also represent a challenge to theory, since, in order to describe the relaxation processes following exposure of polyatomic molecules to ionizing radiation, numerical representations must meet two conflicting requirements. On the one hand, molecular electronic states at short range are best expressed in terms of Gaussian functions. This is because, for polyatomic systems, the polycentric bielectronic integrals associated with interelectronic Coulomb repulsion can be computed much more rapidly within a Gaussian basis set than by using any other basis [10]. Indeed, the use of Gaussian functions is today a de facto standard in all modern quantum-chemistry packages [11]. On the other hand, Gaussian functions are unsuitable to reproduce the characteristic oscillatory behavior of continuum orbitals in the asymptotic radial range, due to the rapid buildup of linear dependencies [12] between the basis elements. Consequently, continuum orbitals obtained numerically from Gaussian functions can only be reliable either in a limited radial domain or within few $\mathrm{eV}$ from threshold opening [13-15]. Nevertheless, Gaussian functions have been rather successful in describing one-photon ionization of molecules to first order of perturbation theory [16-21], since the very localized nature of the ground state effectively restricts the spatial region in which ionization is produced. A similar restriction does not apply to above-threshold ionization (ATI) or to ionization beyond the perturbative regime, thus making the use of Gaussian functions unsuitable for the description

\footnotetext{
*luca.argenti@uam.es
}

of many ultrafast phenomena in the electronic continuum of molecules.

Describing the electronic continuum with Gaussian functions (or any other $L^{2}$-integrable functions) requires the use of specific methods, such as, e.g., the $K$-matrix theory [22-26]. Another popular method is based on the $R$-matrix theory (see, e.g., [27] and references therein), which has been implemented on different $L^{2}$ bases, including Gaussian functions [28-37]. While the $R$-matrix method offers, in principle, considerable flexibility, it also requires the evaluation of truncated electronic integrals, which are not implemented by default in standard quantum-chemistry codes.

The rapid oscillations of continuum orbitals are better described by dedicated radial sets like, e.g., finite-element discrete-variable representations [38-43] and $B$ splines [44-47]. The use of this latter type of function, however, leads to costly polycentric bielectronic integrals or, alternatively, to monocentric expansions which converge too slowly except for the simplest cases (e.g., $\mathrm{H}_{2}$ [48] and hydrides of light elements $[49,50])$. To overcome the limitations of the monocentric expansion common to many basis sets appropriate to represent the continuum, it is possible to expand such basis with a group of polycentric localized functions that are better suited to express concisely the wave function close to the nuclei. Within the single-active-electron approximation, the use of a multicenter $B$-spline basis has proven to be a very effective way to describe arbitrary single-ionization problems [51-61]. Monocentric $B$-spline sets have also been used in association with polycentric Slater-type orbitals $[62,63]$. In the same spirit, monocentric grid representations have been mixed with polycentric Gaussian functions [64-67]. None of these approaches is readily generalized beyond single-active-electron models (e.g., Hartree-Fock and density functional theory), since the functions used to describe the continuum extend across the whole molecule while standard implementations of multielectron techniques cannot handle nonstandard basis sets. This means that any ab initio method has to be implemented from scratch.

A way to circumvent the evaluation of nonstandard polycentric bielectronic integrals is to complement shortrange polycentric Gaussian functions with several diffused 
monocentric Gaussian functions, to cover the midrange radial region, and with a set of $B$ splines, defined starting from a finite radius $R_{0}$, to cover the long-range radial region. If $R_{0}$ is large enough, the overlap between short-range polycentric functions and $B$ splines can be neglected. Furthermore, thanks to the fact that the short-range part of the electronic states is entirely expressed in terms of Gaussian functions, optimized parent-ion and neutral bound states can be computed with standard quantum-chemistry packages. A minimal requirement for this approach to be successful is that the hybrid monocentric basis comprising the diffuse set of Gaussian functions and the $B$ splines (GABSs) can accurately reproduce the scattering observables of a single-particle monocentric problem. In the present work, we show that this is indeed the case. We have tested the performance of the GABS basis for several representative calculations of the interaction of the hydrogen atom with external ionizing radiation, in both perturbative and nonperturbative conditions, either in terms of dipole transition matrix elements or by directly solving the time-dependent Schrödinger equation (TDSE) for realistic ultrashort laser pulses. In order to extend the test of gauge invariance for dipole matrix elements to transitions between continuum states, we have derived two different expressions for the contribution to the transition integrals coming from the radial region beyond the quantization box where the states are numerically computed. We find excellent agreement with accurate numerical calculations based on a purely $B$-spline basis and with the analytical results, when available, in a wide radial and energy range. GABS functions thus look like promising candidates to build unbound molecular electronic states fulfilling all requirements needed to study a wide range of ultrafast phenomena in molecules. Finally, in the comparison between our solutions of the TDSE with spectra available in the literature, for selected ATI processes, we also report fully resolved photoelectron angular distributions and explain their distinctive features in detail.

The article is organized as follows. In Sec. II we introduce the GABS hybrid basis. In Sec. III we quantify the quality of bound and continuum eigenstates of the hydrogen atom obtained with the hybrid basis and of the dipole transition matrix elements between them. In Sec. IV we solve the TDSE at both short (Sec. IV A) and long (Sec. IV B) wavelengths and compare our results with recent ones taken from the literature. In Sec. V we draw our conclusions. Atomic units are used throughout unless stated otherwise.

\section{THEORY AND NUMERICAL METHODS}

\section{A. The GABS basis}

The GABS basis is a monocentric basis comprising a set of Gaussian functions and a set of $B$ splines whose support starts from a given radius $R_{0}$ (see Fig. 1). Due to their fast-descent character, the Gaussian functions are numerically negligible beyond a radius $R_{1}>R_{0}$. Functions expressed in the GABS basis, therefore, are represented by purely Gaussian functions in the short range, i.e., $r<R_{0}$, by a combination of Gaussian and $B$-spline functions in the intermediate region, $R_{0}<r<$ $R_{1}$, and by purely $B$-spline functions thereafter.

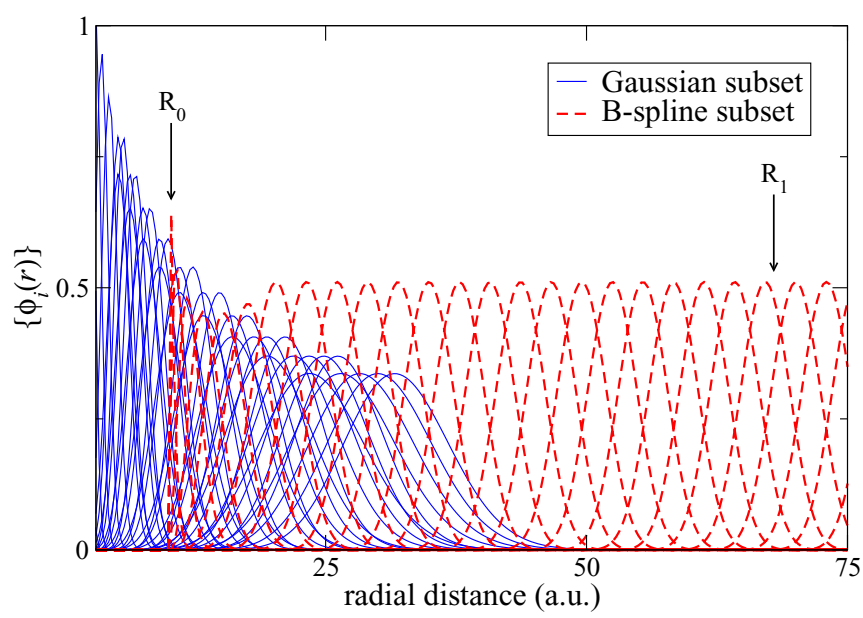

FIG. 1. (Color online) Radial part of Gaussian (solid lines) and $B$-spline (dashed lines) representatives of the monocentric GABS basis as a function of radius. The first $B$-spline node is located at $R_{0}=10$ a.u. The basis defines three characteristic regions: (i) $r \in\left[0, R_{0}\right]$, with only Gaussian functions; (ii) $r \in\left[R_{0}, R_{1}\right]$, Gaussian and $B$-spline functions overlap; (iii) $r \in\left[R_{1}, R_{\mathrm{box}}\right]$, the Gaussian functions are negligible.

The Gaussian spatial basis functions with angular momentum $\ell$ and projection $m$ are defined as

$$
G_{\alpha K}^{\ell m}(\vec{r})=N_{\alpha \ell} \frac{g_{\alpha K}(r)}{r} Y_{\ell m}(\hat{r}),
$$

where

$$
g_{\alpha K}(r)=r^{K+1} e^{-\alpha r^{2}},
$$

$K=\ell, \ell+2, \ell+4, \ldots, \ell \leqslant \ell_{\max }$, and $\ell_{\max }$ is a fixed parameter that defines the maximum angular momentum that the basis can represent. The lower bound on $K$ ensures that $g_{\alpha K}(r) \sim r^{\ell+1}$ is regular at the origin. Finally, $N_{\alpha K}$ is a normalization factor,

$$
u_{\alpha K}(r)=N_{\alpha K} g_{\alpha K}, \quad \int_{0}^{\infty} d r u_{\alpha K}^{2}(r)=1 .
$$

Normally, we use for all orbital angular momenta a single set of even-tempered exponents $\left\{\alpha_{1}, \alpha_{2}, \ldots, \alpha_{N_{\alpha}}\right\}$, generated from the two parameters $\alpha$ and $\beta$ according to the geometric series [13,14],

$$
\alpha_{n}=\alpha \beta^{n-1} .
$$

There are thus $N_{G}^{\ell}=N_{\alpha}\left\lfloor\left(\ell_{\max }-\ell\right) / 2+1\right\rfloor$ such states for each angular momentum $\ell(\lfloor x\rfloor$ is the largest integer $n$ such that $n \leqslant x$ ). Numerically redundant linear combinations must be eliminated from the Gaussian set before it can be used in calculations. To do so, for each orbital angular momentum $\ell$, we diagonalize the overlap matrix $\mathbf{S}_{\alpha K, \beta K^{\prime}}^{\ell}=\left\langle G_{\alpha K}^{\ell 0} \mid G_{\beta K^{\prime}}^{\ell 0}\right\rangle$ and exclude some of the eigenfunctions with the smallest eigenvalues. We refer to the $N_{\mathcal{G}}^{\ell}$ remaining eigenstates as to the preconditioned Gaussian basis $\left\{\left|\mathcal{G}_{1}^{\ell m}\right\rangle,\left|\mathcal{G}_{2}^{\ell m}\right\rangle, \ldots,\left|\mathcal{G}_{N_{\mathcal{G}}^{\ell m}}^{\ell m}\right\rangle\right.$.

$B$ splines are a minimal-support basis for piecewise polynomials of degree $k-1$ that are at least $\mathcal{C}^{k-2}$ in correspondence with a given set of nodes $\left\{t_{i}\right\}_{i=1,2, \ldots, n}$ and $\mathcal{C}^{\infty}$ elsewhere [68]. It can be shown that the support of each $B$ spline is compact 
and covers $k$ consecutive intervals. As a consequence, in this basis, local operators are represented by sparse matrices. This property, which imparts high flexibility to the basis without compromising numerical accuracy, is the major reason why $B$ splines are used for atomic and molecular-physics calculations [44]. In this work we employ $k=7$, which is a good compromise for calculations in double precision.

Preconditioned Gaussian functions and $B$ splines are separately well-conditioned basis sets. When considered together, however, numerical linear dependencies may arise since the two sets overlap over an extended radial region. To prevent numerical instabilities, the spline space is purged from the elements which contribute most to the numerical overcompleteness of the GABS basis. We do so by diagonalizing the projector $\hat{P}_{\mathcal{G}}$ that defines the preconditioned Gaussian space in the basis of $B$ splines (for simplicity, we drop the angular momentum indexes),

$$
\begin{aligned}
\left\langle\mathbf{B}\left|\hat{P}_{\mathcal{G}}\right| \mathbf{B}\right\rangle & =\mathbf{S}_{b g} \mathbf{S}_{g g}^{-1} \mathbf{S}_{g b}=\mathbf{O} \mathbf{\Lambda} \mathbf{O}^{\dagger}, \\
\boldsymbol{\Lambda}_{i j} & =\lambda_{i} \delta_{i j}, \quad \mathbf{O}^{\dagger} \mathbf{O}=\mathbf{1},
\end{aligned}
$$

where

$$
\begin{gathered}
\mathbf{S}_{g g}=\langle\mathcal{G} \mid \mathcal{G}\rangle, \quad \mathbf{S}_{b g}=\langle\mathbf{B} \mid \mathcal{G}\rangle, \quad \mathbf{S}_{g b}=\mathbf{S}_{b g}^{\dagger}, \\
|\mathcal{G}\rangle=\left(\left|\mathcal{G}_{1}\right\rangle,\left|\mathcal{G}_{2}\right\rangle, \ldots,\left|\mathcal{G}_{N_{\mathcal{G}}}\right\rangle\right), \\
|\mathbf{B}\rangle=\left(\left|B_{1}\right\rangle,\left|B_{2}\right\rangle, \ldots,\left|B_{N_{B}}\right\rangle\right) .
\end{gathered}
$$

We exclude from the basis the eigenfunctions whose eigenvalue differs from 1 less than an assigned threshold $\epsilon_{P}^{B}$ (in our case, $\epsilon_{P}^{B}=10^{-8}$ ). The remaining eigenstates form a preconditioned $B$-spline basis that is called $\left\{\left|\mathcal{B}_{1}^{\ell}\right\rangle,\left|\mathcal{B}_{2}^{\ell}\right\rangle, \ldots,\left|\mathcal{B}_{N_{B_{p}}}^{\ell}\right\rangle\right\}$. We use the symbol $\chi_{n}^{\ell}$ to indicate the elements of the resulting regularized GABS basis, $\left\{\chi_{n}^{\ell}\right\}=\left\{\mathcal{G}_{i}^{\ell}\right\} \cup\left\{\mathcal{B}_{j}^{\ell}\right\}$. Although the support of the Gaussian functions is, in principle, the whole $[0, \infty)$ semiaxis, in practice, the Gaussian functions are negligible beyond a certain radius $R_{1}$. As a consequence, the $B$ splines whose support is located beyond $R_{1}$ can be kept unchanged throughout the regularization procedure. This means that, beyond a certain index, the regularized GABS functions are pure $B$ splines. In particular, local operators expressed in this part of the GABS basis retain a sparse character.

\section{B. Spectral resolution of the Hamiltonian}

Different choices for the conditions fulfilled by the state functions at the box boundary lead to different sets of eigenvalues and eigenstates of the Hamiltonian. If the last $B$ spline is excluded, all the functions in residual basis, which we indicate as $\bar{\chi}=\left(\bar{\chi}_{1}, \bar{\chi}_{2}, \ldots\right)$, vanish at the box boundary. When projected in this basis, therefore, the Hamiltonian is Hermitian and corresponds to the case in which the system is confined in a box. Its spectrum comprises only isolated real eigenvalues $\bar{E}_{i}$. The negative-energy box eigenstates $\left|\phi_{i}\right\rangle$ that do not get close to the box boundary approximate the first terms of the Rydberg series of the unconstrained Hamiltonian, while the positive-energy states represent a discrete selection of the generalized continuum eigenstates of the unconstrained
Hamiltonian, up to a certain energy $E_{\max }$, after which the quality of the states starts to deteriorate.

Requiring that the wave function vanishes at the box boundary, however, severely curtails the flexibility of the $B$ splines and of the hybrid basis alike. In particular, such basis reproduces only a limited selection of discretized-continuum states. If the last $B$ spline is present, on the other hand, the basis is able to represent, within the box, any eigenfunction of the Hamiltonian that is regular at the origin, up to $E_{\max }$ [44]. To see this, let us look for an eigenfunction with energy $E$, $\psi_{E}$, in the form

$$
\left|\psi_{E}\right\rangle=|\bar{\chi}\rangle \mathbf{c}_{E}+\left|B_{N}\right\rangle b_{E},
$$

where $B_{N}$ is the last $B$ spline of the basis set. If $E$ belongs to the spectrum of the confined Hamiltonian, the solution is a box eigenstate and the coefficient of the last $B$ spline is zero, $b_{E}=0$. In the other cases, we can derive the other coefficients in terms of $b_{E}$ by solving the $(n-1) \times n$ homogeneous linear system

$$
\left\langle\bar{\chi}|E-H| \psi_{E}\right\rangle=0
$$

which admits the solution

$$
\begin{gathered}
\left|\psi_{E}\right\rangle \propto|\bar{\chi}\rangle \overline{\mathbf{G}}_{0}(E)\left\langle\bar{\chi}|H-E| B_{N}\right\rangle b_{E}+\left|B_{N}\right\rangle b_{E}, \\
\overline{\mathbf{G}}_{0}(E)=\langle\bar{\chi}|E-H| \bar{\chi}\rangle^{-1} .
\end{gathered}
$$

The expression (11) provides valid scattering solutions for any $E \in\left[0, E_{\max }\right]$, which are as accurate as the discretizedcontinuum eigenstates obtained from the diagonalization of the Hamiltonian in the box (a treatment similar to the one presented here can be found, e.g., in [69]). To ensure that the analytic continuation $\Psi_{E}$ of $\psi_{E}$ to the whole $[0, \infty)$ real semiaxis fulfills the condition

$$
\left\langle\Psi_{E} \mid \Psi_{E^{\prime}}\right\rangle=\delta\left(E-E^{\prime}\right),
$$

the value of $b_{E}$ must be determined by matching, at the box boundary, the reduced radial part $u_{\ell E}(r)$ of the wave function,

$$
\psi_{E \ell m}(\vec{r})=\frac{u_{\ell E}(r)}{r} Y_{\ell m}(\hat{r})
$$

( $\ell$ is the formerly implied angular momentum), with a linear combination of the analytical regular $F_{\ell}(\gamma, \rho)$ and irregular $G_{\ell}(\gamma, \rho)$ Coulomb functions with the same energy [70],

$$
u_{\ell E}(r)=\sqrt{\frac{2}{\pi k}}\left[\cos \delta F_{\ell}(\gamma, \rho)+\sin \delta G_{\ell}(\gamma, \rho)\right],
$$

where $k=\sqrt{2 E}, \gamma=-Z / k, \rho=k r, Z$ is the nuclear charge, and $\delta$ is the phase shift. When comparing with ionization experiments, we need to evaluate the transition amplitudes to the detector eigenstates $\psi_{E \hat{\Omega}}^{-}$, i.e., the states that have a well-defined asymptotic energy $E$ and direction $\hat{\Omega}$ in the far future. These are the scattering states which fulfill incoming boundary conditions [71] and have the expression

$$
\psi_{E \hat{\Omega}}^{-}=\sum_{\ell m} \psi_{E \ell m} Y_{\ell m}^{*}(\Omega) e^{-i\left(\sigma_{\ell}+\delta_{\ell}-\ell \pi / 2\right)},
$$

where $\sigma_{\ell}=\arg \Gamma(\ell+1+i \gamma)$ is the Coulomb phase shift [72].

In the case of negative energies, only those solutions to Eq. (11) whose analytical extension is regular at $r \rightarrow \infty$ 
represent valid bound eigenstates. This property can be ensured by requiring that the logarithmic derivative of the radial part of $\psi_{E}$ matches that of the eigenfunction of the Coulomb problem with the same (negative) energy and that is regular at infinity,

$$
\frac{u_{\ell E}^{\prime}(r)}{u_{\ell E}(r)}=\frac{\left[e^{-\rho} \rho^{\ell+1} U(\ell+1-Z / \kappa, 2 \ell+2,2 \rho)\right]^{\prime}}{e^{-\rho} \rho^{\ell+1} U(\ell+1-Z / \kappa, 2 \ell+2,2 \rho)},
$$

where $\kappa=\sqrt{-2 E}, \rho=\kappa r, U(a, b, z)$ is the confluent hypergeometric function of the second kind [70], and both the left-hand side and the right-hand side of (17) are to be evaluated for $r=R_{\text {box }}$ (see Appendix $\mathrm{C}$ for an outline of the derivation).

\section{Dipole transition matrix elements}

Dipole transition matrix elements are a necessary ingredient of both perturbative and nonperturbative radiative transitions. In the next section we compare the reduced dipole transition matrix elements between selected eigenstates $\psi_{E \ell m}$ of the field-free Hamiltonian,

$$
\left\langle\psi_{E \ell}\left\|\mathcal{O}_{1}^{(g)}\right\| \psi_{E^{\prime} \ell^{\prime}}\right\rangle=\sum_{m m^{\prime} \mu} \frac{C_{\ell^{\prime} m^{\prime}, 1 \mu}^{\ell m}}{\sqrt{2 \ell+1}}\left\langle\psi_{E \ell m}\left|\mathcal{O}_{1 \mu}^{(g)}\right| \psi_{E^{\prime} \ell^{\prime} m^{\prime}}\right\rangle,
$$

where $C_{a \alpha, b \beta}^{c \gamma}$ is a Clebsch-Gordan coefficient [73], evaluated in different numerical bases as well as analytically, for three gauges $g$, length, velocity, and acceleration,

$$
\mathcal{O}_{1 \mu}^{(l)}=r_{1 \mu}, \quad \mathcal{O}_{1 \mu}^{(v)}=p_{1 \mu}, \quad \mathcal{O}_{1 \mu}^{(a)}=Z \frac{r_{1 \mu}}{r^{3}} .
$$

The off-shell dipole transition matrix elements in different gauges between physical eigenstates are related to each other through the identities

$$
\begin{gathered}
i\left(E-E^{\prime}\right)\left\langle\psi_{E \ell}\left\|\mathcal{O}_{1}^{(l)}\right\| \psi_{E^{\prime} \ell^{\prime}}\right\rangle=\left\langle\psi_{E \ell}\left\|\mathcal{O}_{1}^{(v)}\right\| \psi_{E^{\prime} \ell^{\prime}}\right\rangle, \\
\left(E-E^{\prime}\right)\left\langle\psi_{E \ell}\left\|\mathcal{O}_{1}^{(v)}\right\| \psi_{E^{\prime} \ell^{\prime}}\right\rangle=i\left\langle\psi_{E \ell}\left\|\mathcal{O}_{1}^{(a)}\right\| \psi_{E^{\prime} \ell^{\prime}}\right\rangle .
\end{gathered}
$$

While the Hamiltonian is particularly sensitive to the rapidly varying parts of the wave function, due to the presence of the kinetic energy operator, the kernels in (19) put a higher emphasis on the wave function at either short or long range. The agreement between matrix elements across different gauges and numerical representations, therefore, is an alternative valuable measure of the wave function quality beyond the numerical fulfillment of the secular equation.

If the continuum-continuum transitions are evaluated between box eigenstates instead of physical states (i.e., if the radial integrals are truncated at $r=R_{\mathrm{box}}$ ), Eq. (21) needs to be modified for what we call the confined-state boundary correction (CBC)

$$
\begin{aligned}
(E & \left.-E^{\prime}\right)\left\langle\left.\psi_{E \ell}\left\|\mathcal{O}_{1}^{(v)}\right\| \psi_{E^{\prime} \ell^{\prime}}\right|_{r \in\left[0, R_{\mathrm{box}}\right]}\right. \\
= & i\left\langle\psi_{E \ell}\left\|\mathcal{O}_{1}^{(a)}\right\| \psi_{E^{\prime} \ell^{\prime}}\right\rangle_{r \in\left[0, R_{\mathrm{box}}\right]} \\
& +\frac{i}{2} \sqrt{2 \ell^{\prime}+1} C_{\ell^{\prime} 0,10}^{\ell 0} \frac{d u_{\ell E}}{d r}\left(R_{\mathrm{box}}\right) \frac{d u_{\ell^{\prime} E^{\prime}}}{d r}\left(R_{\mathrm{box}}\right) .
\end{aligned}
$$

Furthermore, while Eq. (20) remains valid even when considered between box eigenstates, the transition matrix elements themselves do differ from those evaluated between scattering states. In Appendix A we derive and discuss at length the above equations. In the next section, we use them to frame the results of our calculations.

\section{Time-dependent Schrödinger equation}

To compute the effects of intense linearly polarized radiation pulses on a bound state $\phi_{0}$ of a hydrogen atom, we solve the TDSE,

$$
i \partial_{t} \psi(t)=H(t) \psi(t) ; \quad \psi\left(t_{0}\right)=\phi_{1 s},
$$

by expanding the time-dependent wave function $\psi(t)$ in the basis of the box eigenstates of the field-free Hamiltonian, obtained from either the GABS or the purely $B$ spline basis,

$$
\psi(t)=\sum_{i \ell} \phi_{i \ell} c_{i \ell}(t)
$$

where axial symmetry, $m=0$, is assumed throughout. In the present work, beyond the field-free electrostatic term $H_{0}$, the complete Hamiltonian comprises the time-dependent interaction with the external fields $H^{\prime}(t)$ in the dipole approximation,

$$
\begin{gathered}
H(t)=H_{0}+H^{\prime}(t), \\
H_{0}=\frac{p^{2}}{2}-\frac{Z}{r}, \\
H^{\prime}(t)= \begin{cases}\alpha \vec{A}(t) \cdot \vec{p} & \text { velocity gauge, } \\
\vec{E}(t) \cdot \vec{r} & \text { length gauge, }\end{cases}
\end{gathered}
$$

where $\vec{A}(t)$ and $\vec{E}(t)$ are the vector potential and the electric field of the external radiation, respectively. The TDSE is integrated numerically by propagating the solution on a time grid $t_{i}=t_{i-1}+d t$,

$$
\psi(t+d t)=U(t+d t, t) \psi(t),
$$

where $U(t+d t, t)$ is a second-order split exponential propagator,

$$
U(t+d t, t) \equiv e^{-i H_{0} \frac{d t}{2}} e^{-i H^{\prime}\left(t+\frac{d t}{2}\right) d t} e^{-i H_{0} \frac{d t}{2}} .
$$

As the wave function is expressed in a spectral basis, the action on $\psi$ of the two outer exponential factors on the right-hand side of (29) is trivial. The most time-consuming step of the propagation (28) is the evaluation of $\exp \left[-i H^{\prime}(t+d t / 2) d t\right]$ on $\psi$. Here we carry out this step by representing the exponential operator on the truncated Krylov basis generated by the repeated action of $H^{\prime}(t+d t / 2)$ on the $\exp \left[-H_{0} d t / 2\right] \psi(t)$ state. Under the conditions examined in the present work, where fields with only moderate intensities are contemplated, this representation converges rapidly with respect to the size $N_{K}$ of the Krylov space, which can generally be truncated to $N_{K} \leqslant 5$. Such a fast convergence is a consequence of the factorization between the $H_{0}$ and the $H^{\prime}$ action achieved with the splitting in (29).

The bound-state population $P_{n \ell}$ and the asymptotic energyresolved and angularly resolved photoelectron distribution $d^{2} P(E, \cos \theta) / d E d \cos \theta$ are obtained by projecting the wave packet, at any time after the external pulse is over, on a complete set of bound and scattering eigenstates of the fieldfree Hamiltonian, computed with the techniques described 
TABLE I. Energies for the first six bound $s$ and $p$ hydrogen states, obtained using the $B$-spline basis and two purely Gaussian basis.

\begin{tabular}{lllrlr}
\hline \hline & & $\begin{array}{c}B \text { splines } \\
\ell=0,1\end{array}$ & \multicolumn{2}{c}{$\begin{array}{c}\text { Gaussian }\left(\ell_{\max }=20\right) \\
\text { Gaussian }\left(\ell_{\max }=2\right) \\
\ell=0\end{array}$} \\
\hline 1 & Analytical & -0.50000000 & -0.49996942 & -0.49993963 \\
2 & -0.5 & -0.12500000 & -0.12499618 & -0.12500000 & -0.12499244 \\
3 & -0.125 & -0.05555556 & -0.05555436 & -0.05555552 & -0.05525990 \\
4 & $-0.0(5)$ & -0.03125000 & -0.03110496 & -0.03111820 & -0.01909004 \\
5 & -0.03125 & -0.02000000 & -0.01593253 & -0.01612874 & 0.04060086 \\
6 & -0.02 & -0.01388889 & 0.00422693 & 0.00368341 & 0.14949865 \\
\hline \hline
\end{tabular}

in Sec. II B,

$$
\begin{gathered}
P_{n \ell}=\left|\left\langle\psi_{n \ell 0} \mid \psi(t)\right\rangle\right|^{2}, \\
\frac{d^{2} P(E, \cos \theta)}{d E d \cos \theta}=2 \pi\left|\left\langle\psi_{E, \hat{\Omega}}^{-} \mid \psi(t)\right\rangle\right|^{2} .
\end{gathered}
$$

\section{VALIDATION OF THE GABS BASIS}

\section{A. Bound and continuum states of hydrogen}

In this section we illustrate the accuracy of the GABS basis from the perspective of the spectral resolution of the field-free electrostatic Hamiltonian of hydrogen by comparing the results with the analytical ones and with those obtained using both a purely $B$-spline basis and a purely Gaussian basis. We use $B$ splines of degree $k=7$ defined on a uniform grid with node spacing $\Delta r=0.5$ a.u. up to a maximum radius $R_{\mathrm{box}}=$ 1000 a.u. and $N_{\alpha}=22$ Gaussian exponents $\alpha_{n}=\alpha \beta^{n-1}$ for the even-tempered Gaussian basis, with $\alpha=0.01, \beta=1.46$. In Table I, we report the energies for the bound $s$ and $p$ hydrogen states with principal quantum number $n \leqslant 6$, obtained by using two different values of $\ell_{\max }, \ell_{\max }=2$ and $\ell_{\max }=20$. As anticipated, $B$ splines provide essentially exact results for all the states considered, while the quality of the eigenvalues obtained with the Gaussian functions deteriorates already for $n=4$, due to their inability to reproduce the many oscillations that Rydberg satellites have. Extending $\ell_{\max }$ from 2 to 20 improves significantly the energy of the third and fourth states. Even with the larger even-tempered basis, however, the results still remain well off the mark from the $n=5$ state on. In the calculation with the Gaussian functions, the error on the energy of the ground state is one order of magnitude larger than for the first excited state. This circumstance is peculiar to $s$ orbitals and is due to the well-known difficulty Gaussian functions have in reproducing the cusp condition at the origin.
Indeed, the energies of the $2 p$ and $3 p$ states reported in Table I are two orders of magnitude more accurate than those of the $2 s$ and $3 s$ ones computed with the same basis. Table II reports the energy of several bound hydrogen states computed with three GABS bases that differ for the value of $\ell_{\max }$ and for the position of the first $B$-spline node (a more extensive compilation of states is given in the Supplemental Material [74]). In $s$ symmetry, all three GABS bases give excellent values across the bound spectrum with errors, for $n \geqslant 2$, of the order of $\sim 10^{-6}$ or lower, irrespective of $\ell_{\max }$. The energy of all the $p$ orbitals is accurate to eight decimal digits. We can better understand the origin of GABS good performance by looking at the contribution of $B$ splines and Gaussian functions to the radial part of the eigenstates computed with the hybrid basis. Figure 2 shows these quantities for the $7 s$ and $15 s$ states, with the corresponding radial orbitals obtained with the $B$-spline basis as a reference. The plots in Fig. 2 illustrate two distinctive features of the GABS basis. First, $B$ splines complete the Gaussian basis in the outer region. As a result, the basis can represent the oscillatory behavior of highly excited bound states there. Second, $B$ splines effectively counterbalance any value that the diffuse Gaussian functions can take in the intermediate region. As a result, the diffuse Gaussian functions acquire larger flexibility in the inner region as well.

The conclusions above also apply to the states in the continuum. In Fig. 3(a), we compare the radial part of the analytical $\psi_{s E}$ generalized eigenfunction for $E=0.1$ a.u. with the corresponding numerical quantity computed with the unconstrained-continuum method described in Sec. II using the GABS basis. The two curves are indistinguishable to the naked eye. We quantify the numerical error in Fig. 3(b) for three different energies: $E=0.1,1$, and 4 a.u. In the outer radial region ( $r \geqslant 55$ a.u.), where only $B$ splines contribute significantly to the wave function, the numerical error is of the order of $10^{-10}, 10^{-7}$, and $10^{-4}$ for the three considered

\begin{tabular}{|c|c|c|c|c|c|}
\hline \multirow[b]{2}{*}{$n$} & \multirow[b]{2}{*}{ Analytical } & \multicolumn{2}{|c|}{$\ell_{\max }=20, R_{0}=10$ a.u. } & \multicolumn{2}{|c|}{$\ell_{\max }=2, \ell=0$} \\
\hline & & $\ell=0$ & $\ell=1$ & $R_{0}=5$ a.u. & $R_{0}=10$ a.u. \\
\hline 1 & -0.5 & -0.49997191 & & -0.49994231 & -0.49994092 \\
\hline 2 & -0.125 & -0.12499648 & -0.12500000 & -0.12499278 & -0.12499261 \\
\hline 3 & $-0.0(5)$ & -0.05555451 & -0.05555556 & -0.05555241 & -0.05555337 \\
\hline 4 & -0.03125 & -0.31249561 & -0.03125000 & -0.03124909 & -0.03124907 \\
\hline 5 & -0.02 & -0.01999977 & -0.02000000 & -0.01999954 & -0.01999952 \\
\hline 6 & $-0.013(8)$ & -0.01388876 & -0.01388889 & -0.01388862 & -0.01388861 \\
\hline
\end{tabular}

TABLE II. Energies for the first $6 s$ and $5 p$ bound hydrogen states obtained with three and one GABS bases, respectively. 


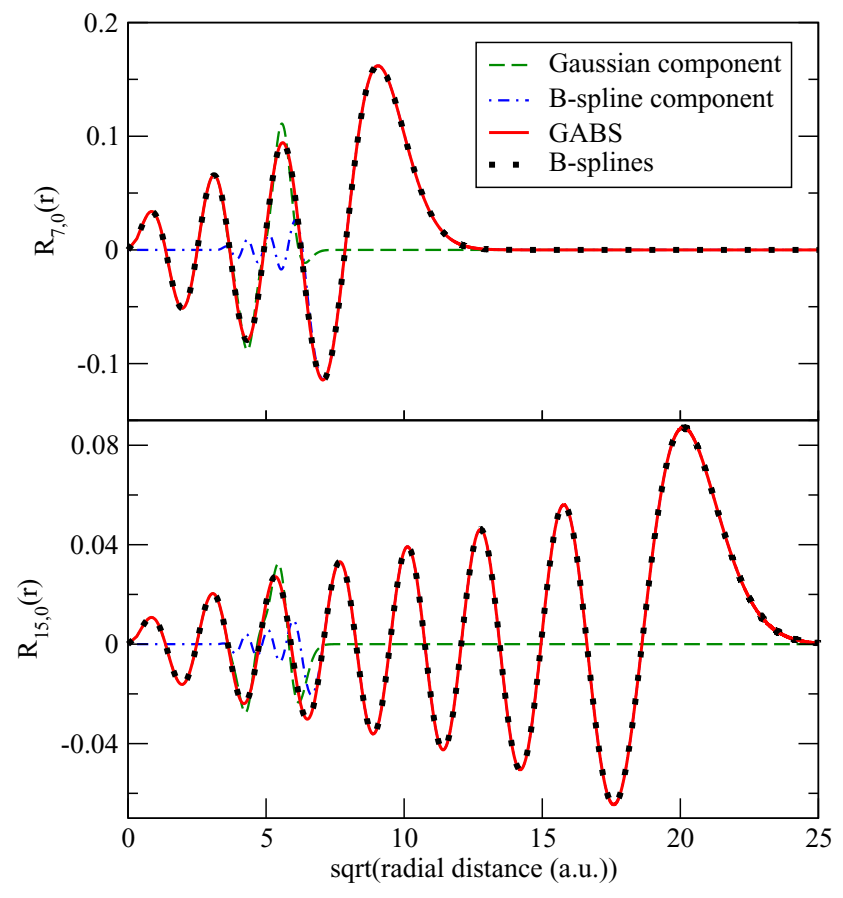

FIG. 2. (Color online) Radial part of the $7 s$ (top panel) and $15 s$ (bottom panel) hydrogen Rydberg states as a function of $\sqrt{r}$, computed with $B$ splines (dots) and GABS (solid line). For the latter case, the Gaussian component (dashed line) and the $B$-spline component (dash-dotted line) are separately shown.

energies, respectively. In the intermediate radial region, between $\simeq 20$ and $\simeq 40$ a.u., where both the diffuse Gaussian functions and the $B$ splines contribute to the wave function, the absolute representation error has a plateau of the order of $10^{-7}$ for $E \leqslant 1$ a.u. At higher energies the error is dominated by the larger $B$-spline asymptotic error. Finally, in the shortrange region, where the wave function is represented only in terms of Gaussian functions, for the two smallest energies the absolute error has a plateau of the order of $10^{-4}$, i.e., comparable to the asymptotic $B$-spline representation error for $E=4$ a.u. (see Supplemental Material [74] for an analysis of the quality of continuum wave functions based on the phase shift).

\section{B. Radiative transitions}

Beyond energy, the other observable needed to describe the time evolution of a system under the influence of external fields is the dipole operator. It is essential, therefore, to assess the accuracy of the dipole matrix elements in the hybrid basis. In Table III we compare the analytical reduced matrix elements $\left|\left\langle\psi\left\|p_{1}\right\| \psi^{\prime}\right\rangle\right|$ for a few selected dipole transitions between bound hydrogenic states with those obtained numerically with $B$ splines, with GABS functions in the three gauges of length, velocity, and acceleration, as well as, in velocity gauge, for two additional representative pairs of the $\ell_{\max }$ and $R_{0}$ parameters. The transitions matrix elements computed with the $B$ splines are exact for all six tabulated decimal digits.

In Fig. 4 we compare the absolute value of some selected bound-continuum reduced dipole matrix elements in velocity gauge, obtained using the GABS basis, with the analytical

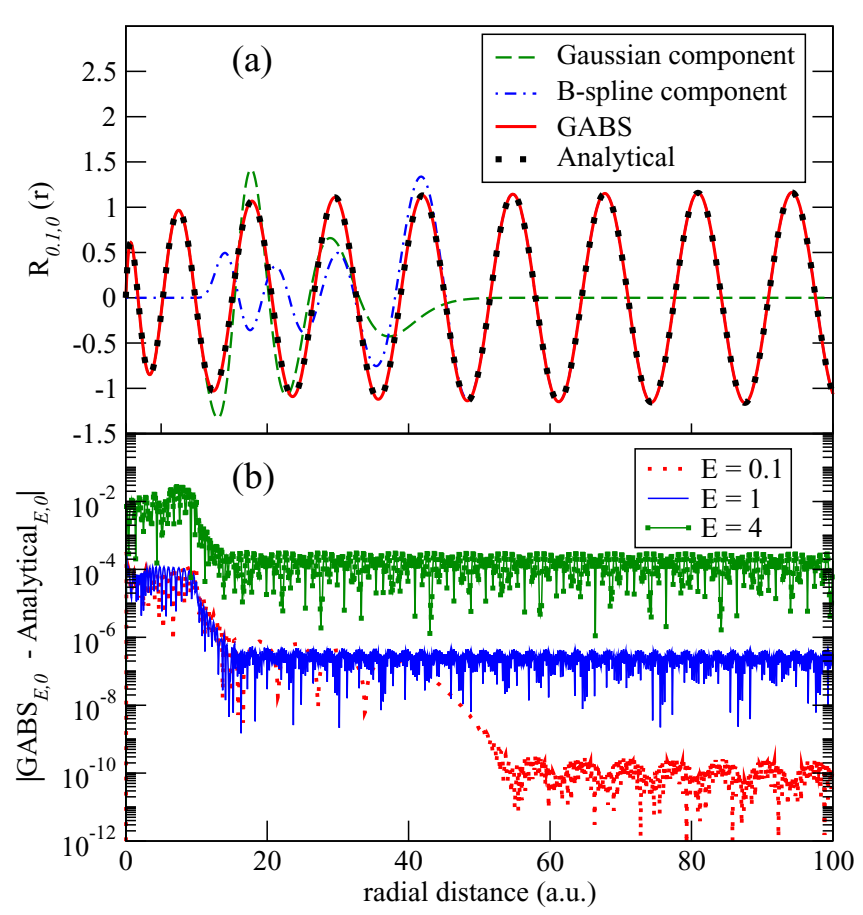

FIG. 3. (Color online) (Top panel) Radial part of the $\psi_{s E}$ hydrogen scattering state with $E=0.1$ a.u., computed analytically (dots) and numerically using GABS (solid line) $R_{0}=10$ a.u., $\ell_{\max }=20$. The Gaussian (dashed line) and the $B$ spline (dash-dotted line) components of the numerical function are also shown. (Bottom panel) Absolute local error of the scattering states $\psi_{s E}$ computed with GABS for $E=0.1$ a.u. (dots), $E=1$ a.u (solid line), and $E=4$ a.u. (dotted line). See text for more details.

results (see Appendix C). The agreement is generally very good, with errors of the order of 1 part in 10000 up to $E=$ 2 a.u. At low energies, for the $p$ - $d$ transitions the errors are smaller than 1 part in $10^{6}$ up to $E=1$ a.u. With the current choice of parameters, the error in the matrix element starts to increase progressively above $E=2$ a.u., reaching the level of a few percent for $E=5$ a.u. The agreement between the dipole matrix elements in length, velocity, and acceleration gauge is illustrated in the representative case of the transition from the $1 s$ orbital in Fig. 5.

In the dipole matrix elements examined so far, at least one of the wave functions is localized near the origin. To be able to represent multiphoton transitions and nonperturbative effects, however, accurate continuum-continuum matrix elements are needed as well. In Fig. 6 we compare the analytical results for $\left|\left\langle\psi_{\ell E}\left\|p_{1}\right\| \psi_{\ell^{\prime} E^{\prime}}\right\rangle\right|$ (see Appendix C and Ref. [75]) with those obtained numerically using the GABS basis in the length, velocity, and acceleration gauges, as a function of the final discretized-continuum energy index, for three representative initial discretized-continuum states with $E \simeq 1,2$, and 3 a.u. A few comments are in order. First, since the integration domain of the numerical transition integrals is limited to the box, the length and the velocity gauge agree with each other but differ qualitatively from the acceleration matrix element. On the other hand, the latter gauge, which has a fast decreasing kernel, is in excellent agreement with the analytical result. Furthermore, while in length and velocity 
TABLE III. Several bound-bound radiative transitions obtained via the analytical formula, using the $B$-spline basis and the GABS bases.

\begin{tabular}{|c|c|c|c|c|c|c|c|}
\hline \multirow[b]{2}{*}{ Transition } & \multirow[b]{2}{*}{ Analytical } & \multirow[b]{2}{*}{$B$ splines } & \multicolumn{3}{|c|}{ GABS $_{1}{ }^{a}$} & \multirow{2}{*}{$\begin{array}{l}\mathrm{GABS}_{2}{ }^{\mathrm{b}} \\
\text { Velocity }\end{array}$} & \multirow{2}{*}{$\begin{array}{l}\mathrm{GABS}_{3}{ }^{\mathrm{c}} \\
\text { Velocity }\end{array}$} \\
\hline & & & Length & Velocity & Acceleration & & \\
\hline $1 s-2 p$ & 0.483850 & 0.483850 & 0.483859 & 0.483855 & 0.483852 & 0.483860 & 0.483860 \\
\hline $2 s-3 p$ & 0.212834 & 0.212834 & 0.212847 & 0.212844 & 0.212843 & 0.212855 & 0.212855 \\
\hline $3 s-4 p$ & 0.132935 & 0.132935 & 0.132946 & 0.132943 & 0.132945 & 0.132952 & 0.132952 \\
\hline $2 p-3 d$ & 0.466297 & 0.466297 & 0.466297 & 0.466297 & 0.466297 & 0.466297 & 0.466297 \\
\hline $3 p-4 d$ & 0.260048 & 0.260048 & 0.260048 & 0.260048 & 0.260048 & 0.260048 & 0.260048 \\
\hline $3 d-4 f$ & 0.430680 & 0.430680 & 0.430680 & 0.430680 & 0.430680 & & \\
\hline
\end{tabular}

${ }^{\mathrm{a}} \ell_{\max }=20, R_{0}=10$.

${ }^{\mathrm{b}} \ell_{\max }=2, R_{0}=5$.

${ }^{\mathrm{c}} \ell_{\max }=2, R_{0}=10$.

gauge the matrix elements oscillate wildly with respect to either energy indexes, the matrix element in the acceleration gauge is a smooth function, except for the on-shell cusp. The discrepancy between length and velocity, on the one side, and acceleration, on the other side, is readily explained: We are neglecting the contribution to the integral of the part of the wave function outside the quantization box (see Appendices A and B). Indeed, the oscillations in, say, the velocity gauge matrix element can be shown to depend on the size of the quantization box. In fact, as the size of the box is increased, the velocity matrix elements are expected to converge weakly to the acceleration value (i.e., their integrals with any smooth test functions converge to the same value as $R_{\text {box }} \rightarrow \infty$ ).

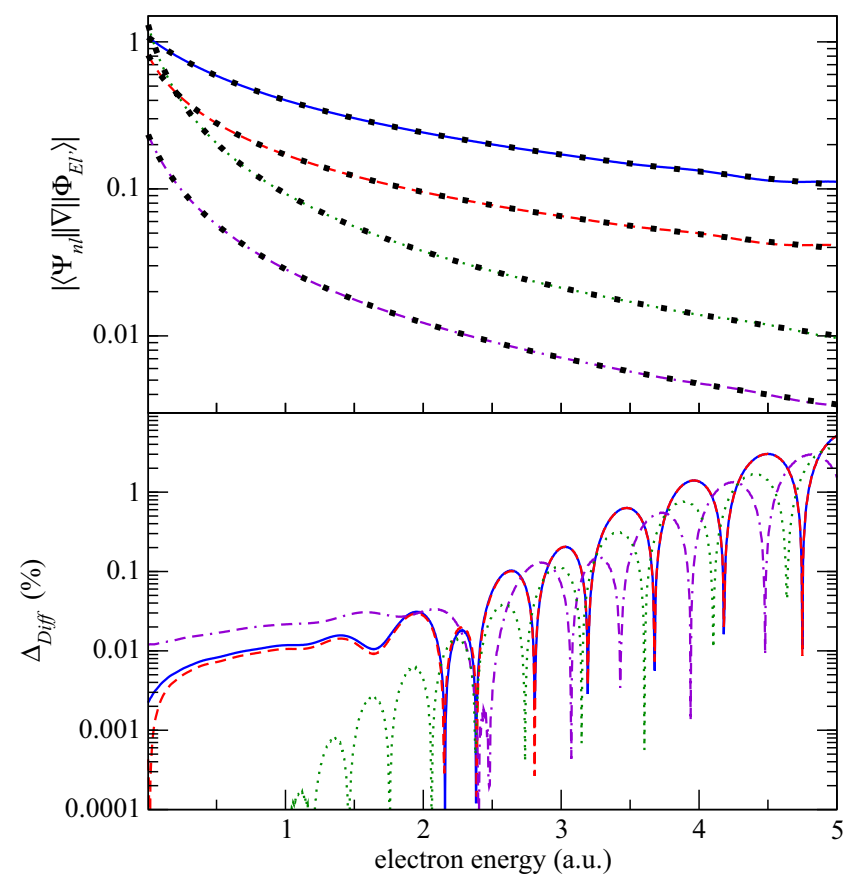

FIG. 4. (Color online) (Top panel) Absolute value of selected reduced dipole transition matrix elements in velocity gauge computed using the GABS basis and compared with the corresponding analytical results (solid squares). (Bottom panel) Relative percent deviation between numerical and analytical results. The following transitions are shown: $1 s-\epsilon_{p}$ (solid line), $2 s-\epsilon_{p}$ (dashed line), $2 p-\epsilon_{s}$ (dash-dotted line), $2 p-\epsilon_{d}$ (dotted line).
The acceleration gauge does not suffer from this dependence thanks to the fact that its kernel decreases rapidly with the electronic radius. The three gauges can thus be reconciled by adding to the length and velocity matrix elements the contribution coming from the region outside the quantization box, which in Appendix A we show to be expressible in the form of a confined-boundary correction [see Eq. (A17)]. This is what we have done in Fig. 7 for the illustrative case of the velocity gauge. As the picture shows, the outer contribution to the matrix elements exactly cancels the original fast oscillations, leading to a smooth matrix element that is in excellent agreement with the analytical result. In Appendix D we derive an approximated correction for the outer integral which applies for arbitrary initial and final energies.

Notice that, as shown in [76], inclusion of the contribution from outside the box is essential to correctly evaluate abovethreshold multiphoton ionization cross sections in length and velocity gauges in the framework of perturbation theory. When seeking solutions of the TDSE, on the other hand, inclusion of the contributions from outside the box is not necessary provided that all continuum-continuum matrix elements are consistently evaluated in the same box and the electronic wave packet does not reach the boundaries of the box before

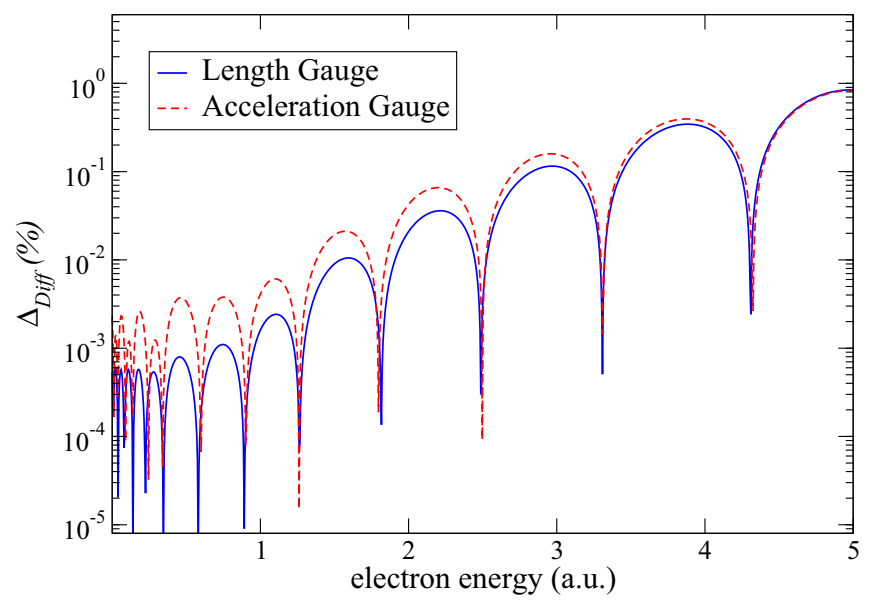

FIG. 5. (Color online) Relative percent deviation of $\left|\left(E-E_{1 s}\right)\left\langle\psi_{E_{p}}\left\|\mathcal{O}_{1}^{(l)}\right\| \psi_{1 s}\right\rangle\right| \quad$ and $\quad\left|\left(E-E_{1 s}\right)^{-1}\left\langle\psi_{E_{p}}\left\|\mathcal{O}_{1}^{(a)}\right\| \psi_{1 s}\right\rangle\right|$ from $\left|\left\langle\psi_{E_{p}}\left\|\mathcal{O}_{1}^{(v)}\right\| \psi_{1 s}\right\rangle\right|$ (solid line and dashed line, respectively), computed with a GABS basis using $R_{0}=10$ a.u., $\ell_{\max }=20$. 


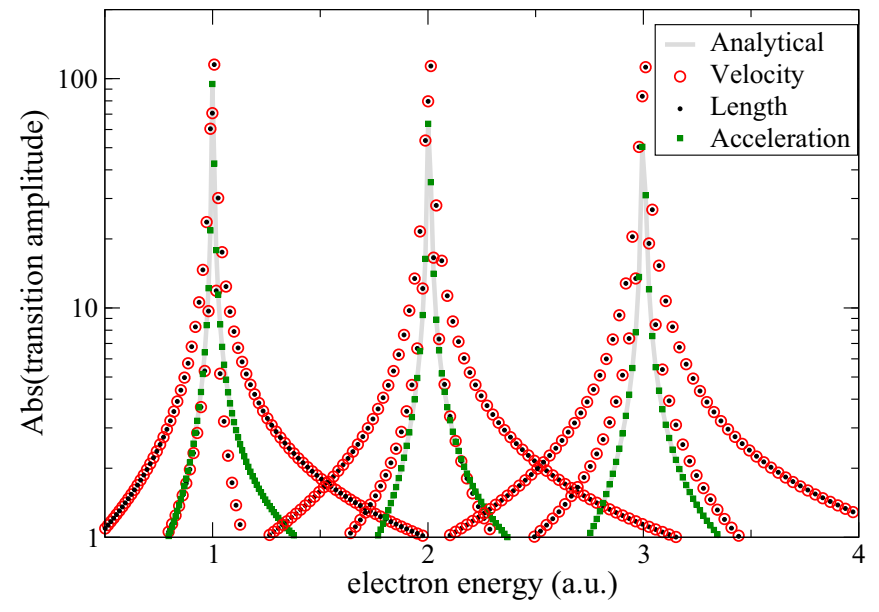

FIG. 6. (Color online) Absolute value of the reduced transition matrix elements $\left(E_{p}-E_{s}\right)\left\langle\psi_{E_{p}}\left\|\mathcal{O}_{1}^{l}\right\| \psi_{E_{s}}\right\rangle$ (black dots), $\left\langle\psi_{E_{p}}\left\|\mathcal{O}_{1}^{v}\right\| \psi_{E_{s}}\right\rangle$ (red circles), $\left(E_{p}-E_{s}\right)^{-1}\left\langle\psi_{E_{p}}\left\|\mathcal{O}_{1}^{a}\right\| \psi_{E_{s}}\right\rangle$ (green solid squares), computed with GABS for several scattering states by truncating the radial integral at $R_{\text {box }}$. Three representative energies for the $s$ state are shown: 1 a.u. (first peak), 2 a.u. (second peak), and 3 a.u. (third peak). The numerical results are compared with the exact analytical result in velocity gauge (gray solid line).

projection onto physical eigenstates is performed (see, e.g., the discussion in [77]).

\section{TIME-DEPENDENT RESULTS}

The ultimate test of the viability of the GABS basis for the description of laser-driven processes is to use it to reproduce fully differential photoelectron observables in nonperturbative

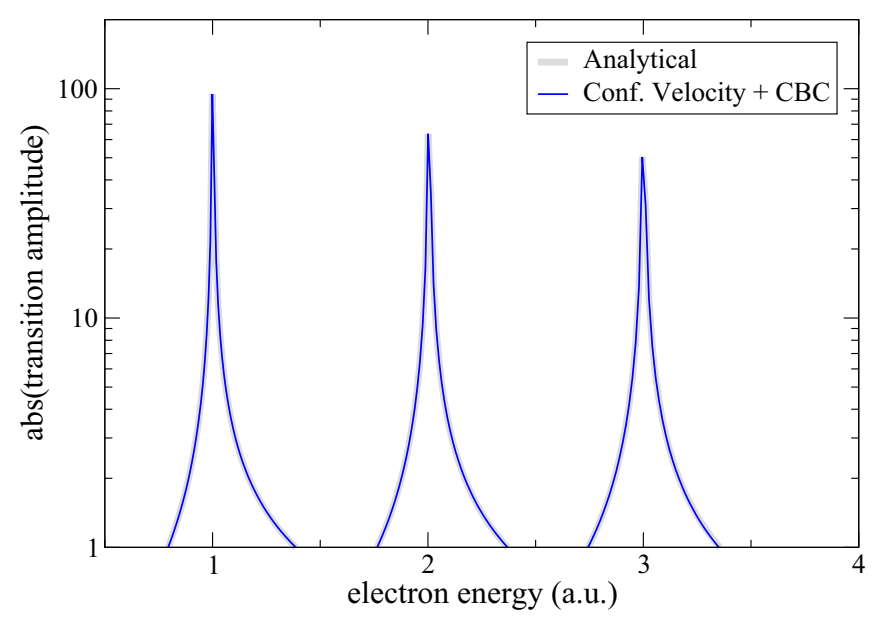

FIG. 7. (Color online) Absolute value of the reduced velocitygauge hydrogen dipole matrix element $\left\langle\psi_{E_{p}}\left\|\mathcal{O}_{1}^{v}\right\| \psi_{E_{s}}\right\rangle$ from three $s$ scattering states $(E=1,2,3$ a.u.) to several $p$ scattering states evaluated as the sum of two contributions (thin solid line). The first contribution is the numerical radial integral shown in Fig. 6, which is computed with the GABS basis and truncated at $R_{\mathrm{box}}$, and the second contribution is the CBC term derived in Appendix A. The agreement between the numerical result and the exact analytical result (thick solid line) is excellent. conditions by solving the TDSE numerically. In this section, we examine the photoelectron distribution for the ATI [78] of the hydrogen atom from the ground state under the influence of single, intense, comparatively long ultraviolet pulses,

$$
\mathrm{H}(1 s)+n \gamma_{\mathrm{Uv}} \rightarrow \mathrm{H}^{+}+e_{\vec{k}}^{-},
$$

for which recent independent data to compare with are available. The methodology used to solve numerically the TDSE and to extract the asymptotic differential photoelectron observables at the end of the interaction was described in Sec. II D. We conduct the simulation using a purely $B$-spline basis, which is known to provide good reference results [79], as well as a GABS basis, with $R_{0}=10$ a.u. and $\ell_{\max }=20$. To assess the accuracy of the solution, we use the same laser parameters employed in two recent works, one by Rodríguez et al. [80] ( $\omega=0.35$ a.u., $I=1.4 \times 10^{13} \mathrm{~W} / \mathrm{cm}^{2}$, 30 -cycle $\cos ^{2}$-envelope electric laser pulse) and the other one by Grum-Grzhimailo et al. [81] ( $\omega=0.114$ a.u., $I=$ $10^{14} \mathrm{~W} / \mathrm{cm}^{2}, 20$-cycle $\cos ^{2}$-envelope electric laser pulse). When reproducing these two simulations, the angular expansion of the wave function is truncated at $\ell=10$ and $\ell=20$, respectively. It should be noted that we conduct the simulation in velocity gauge starting from the definition of the external vector potential as

$$
\vec{A}(t)=\hat{z} A_{0} \cos ^{2}\left(\frac{\pi t}{\tau}\right) \cos (\omega t+\varphi) \theta(\tau / 2-|t|),
$$

where $\varphi$ is the carrier-envelope phase (CEP), $\tau$ is the pulse duration (twice the full width at half maximum of the intensity profile), and $\theta(x)$ is the Heaviside step function. This way, the external electric field, given by $\vec{E}=-c^{-1} \partial_{t} \vec{A}(t)$, automatically integrates to zero, as it should [82]. On the other hand, in [80] a parametrization for the electric field similar to (33) is used instead,

$$
\vec{E}(t)=\hat{z} E_{0} \cos ^{2}\left(\frac{\pi t}{\tau}\right) \sin (\omega t) \theta(\tau / 2-|t|) .
$$

This latter expression differs from the one that can be derived from our definition (33) of the vector potential, due to the time dependence of the pulse envelope. Furthermore, Eq. (34) is applicable, in principle, only for zero CEP, since the field must integrate to zero [82]. Having said that, for long pulses such as those considered in this and their work (20-30 cycles), the difference between the two parametrizations is negligible and affects only minor details of the photoelectron spectra.

\section{A. Short wavelength}

In our first simulation, which reproduces the results by Rodríguez et al., the laser angular frequency is $\omega=0.35$ a.u. Figure 8 compares the ATI spectra obtained using GABS and $B$ splines with the corresponding quantity digitized from [80]. The spectra obtained with GABS and $B$ splines are in excellent agreement down to the tiniest detail, indicating that the solution of the TDSE is accurate. The main peaks are in excellent agreement also with the results from the literature. Minor departures between the current prediction and the literature are visible only in the background signal, which is six orders of magnitude smaller than the dominant peak signal. We attribute these discrepancies to the different parametrizations (33) 


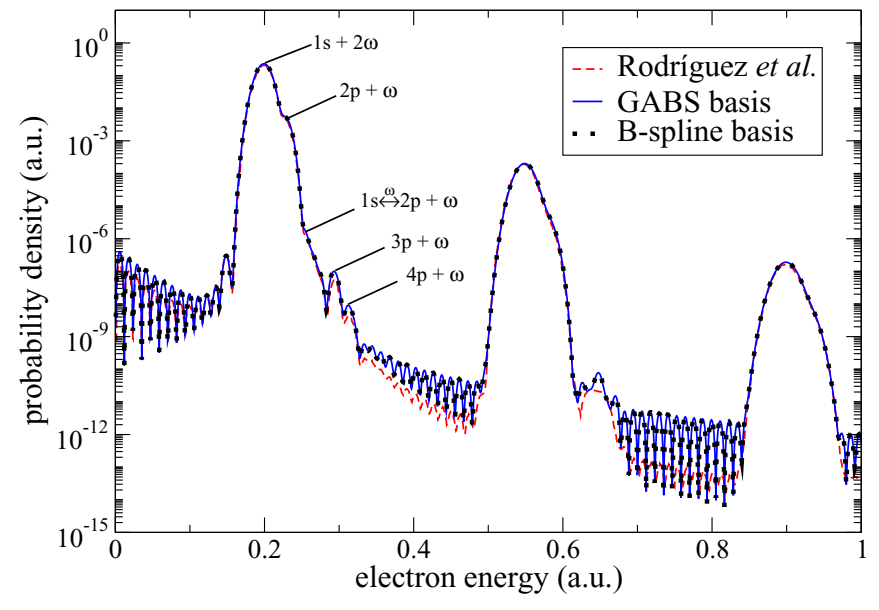

FIG. 8. (Color online) ATI photoelectron spectrum from hydrogen ground state due to a 30-cycle $\cos ^{2}$ laser pulse with $\omega=0.35$ a.u. and $I=14 \mathrm{TW} / \mathrm{cm}^{2}$. The present results, computed by solving the TDSE using either a purely $B$-spline basis (dots) or the GABS basis (solid line), are compared with the spectrum obtained under similar conditions by Rodríguez et al. [80]. See text for more details.

and (34) of the external pulse employed in the two simulations. As anticipated, these differences are entirely negligible to all practical purposes. At least two photons are needed to ionize the atom, with the first excitation step being close to the $1 s-2 p$ resonance. As a consequence, the ATI process is resonantly enhanced, resulting in secondary peaks in the ATI spectrum, as discussed in [80]. In particular, the first shoulder that is visible at $E=0.225$ a.u., on the upper-energy side of the first ATI peak, and the two peaks at $E \simeq 0.29$ a.u. and $E \simeq 0.32$ a.u. are due to the components of the two-photon transition that are resonant with the $2 p, 3 p$, and $4 p$ states, respectively. This can be realized by looking at the finite-pulse version of the lowest-order two-photon transition amplitude for this process,

$$
\begin{aligned}
\mathcal{A}_{E \ell \leftarrow 1 s}^{(2)}= & -i \int d \omega \tilde{F}(-\omega) \tilde{F}\left(E_{1 s}+\omega-E\right) \\
& \times\left\langle E \ell\left|\mathcal{O} G_{0}^{+}\left(E_{1 s}+\omega\right) \mathcal{O}\right| 1 s\right\rangle,
\end{aligned}
$$

where

$$
\tilde{F}(\omega)=\mathscr{F}[F](\omega)=\frac{1}{\sqrt{2 \pi}} \int d t F(t) e^{-i \omega t},
$$

$\mathcal{O}$ is the transition operator $\epsilon_{1}^{\mu} \mathcal{O}_{1 \mu}^{(g)}$ in either the length $(g=l)$ or the velocity $(g=v)$ gauge, and $F(t)$ the corresponding $E(t)$ or $\alpha A(t)$ fields along the polarization direction $\hat{\epsilon}=\epsilon_{1}^{\mu} \hat{x}_{1 \mu}$ (for fields linearly polarized along the $z$ axis, $\epsilon_{1}^{\mu}=\delta_{\mu 0}$, the projection of the angular momentum remains zero, $m=0$ ). In contrast to the equivalent expression for monochromatic fields, Eq. (35) is valid for nonresonant as well as for resonant transitions, provided that Rabi oscillations, ac-Stark shifts and depletion of the ground-state population can be neglected. In the present case, the laser central frequency $\omega=0.35$ a.u. is detuned with respect to the $1 s-2 p$ transition by $\delta=\omega-\omega_{2 p 1 s}=-0.025$ a.u. (we introduce the notation $\left.\omega_{i j}=E_{i}-E_{j}\right)$. At peak intensity $F_{0}$, the variation of the ground-state population $\Delta P_{1 s}$ due to Rabi oscillations is $\Delta P_{1 s}=\left[1+\left(\delta / \Omega_{0}\right)^{2}\right]^{-1}=0.26$, where $\Omega_{0}=\left|F_{0} \mathcal{O}_{2 p, 1 s}\right| \simeq$
0.015 a.u. Therefore, the system remains mostly in the ground state; hence, Eq. (35) is expected to provide meaningful guidance for a qualitative interpretation of the first group of peaks in the ATI spectrum. Quantitative predictions, of course, require a separate numerical solution of the TDSE for at least the $1 s$ and the $n p$ essential states [80]. Indeed, the population transfer between the $1 s$ and the $2 p$ states is small but not negligible. Furthermore, the effective Rabi period $T_{\Omega}=2 \pi / \sqrt{\delta^{2}+\Omega_{0}^{2}}$, which is strongly modulated across the laser pulse, is comparable to the duration of the pulse itself. As a consequence, the amplitude of the $2 p$ state undergoes a sharp isolated variation, which manifests in the spectrum as a broad feature around the resonant $2 p$ peak, rather than in the form of the Autler-Townes doublet that characterizes complete Rabi oscillations.

Keeping in mind these caveats, let us go back to Eq. (35). To disentangle the resonant component from the nonresonant one, we split the retarded resolvent in an off-shell part $G_{0}^{P}$ and an on-shell part,

$$
\begin{aligned}
& G_{0}^{+}(\omega)=G_{0}^{P}(\omega)-i \pi \delta\left(\omega-H_{0}\right), \\
& G_{0}^{P}(\omega)=\mathrm{P} \frac{1}{\omega-H_{0}} .
\end{aligned}
$$

The two-photon transition amplitude (35) then splits into a corresponding principal-value term $\mathcal{A}_{E \leftarrow 1 s}^{(2, P)}$ and an on-shell term $\mathcal{A}_{E \leftarrow 1 s}^{(2, r)}$,

$$
\mathcal{A}_{E \leftarrow 1 s}^{(2)}=\mathcal{A}_{E \leftarrow 1 s}^{(2, P)}+\mathcal{A}_{E \leftarrow 1 s}^{(2, r)} .
$$

The principal-value component of the transition amplitude,

$$
\begin{aligned}
\mathcal{A}_{E \ell \leftarrow 1 s}^{(2, P)}= & -i \int d \omega \tilde{F}(-\omega) \tilde{F}\left(E_{1 s}+\omega-E\right) \\
& \times\left\langle E \ell\left|\mathcal{O} G_{0}^{P}\left(E_{1 s}+\omega\right) \mathcal{O}\right| 1 s\right\rangle,
\end{aligned}
$$

is responsible for the main peak in Fig. 8 at $E=0.2$ a.u. This is obvious for the contribution of the virtual states, i.e., those $|\epsilon p\rangle$ states in the expansion of $G_{0}^{P}\left(E_{1 s}+\omega\right)$ whose energy $\epsilon$ is far from $E_{1 s}+\omega_{0}$, where $\omega_{0}$ is the central angular frequency of the pulse,

$$
\begin{aligned}
P & \int d \omega \tilde{F}(-\omega) \tilde{F}\left(E_{1 s}+\omega-E\right) \frac{\mathcal{O}_{E \ell, \epsilon p} \mathcal{O}_{\epsilon p, 1 s}}{E_{1 s}+\omega-\epsilon} \\
& \simeq \frac{\mathcal{O}_{E \ell, \epsilon p} \mathcal{O}_{\epsilon p, 1 s}}{E_{1 s}+\omega_{0}-\epsilon} \int d \omega \tilde{F}(-\omega) \tilde{F}\left(E_{1 s}+\omega-E\right) \\
& =\frac{\mathcal{O}_{E \ell, \epsilon p} \mathcal{O}_{\epsilon p, 1 s}}{E_{1 s}+\omega_{0}-\epsilon} \sqrt{2 \pi} \mathscr{F}\left[F^{2}\right]\left(E_{1 s}-E\right) .
\end{aligned}
$$

Though less evident, the same is true for the asymmetric principal-value contribution of the nonvirtual excitations. Numerical tests show that the characteristic positions and widths of the latter are comparable to those of the virtual contributions.

The picture qualitatively changes for the on-shell contribution to the transition amplitude $\mathcal{A}_{E \leftarrow 1 s}^{(2, r)}$. Each term in the eigenstate expansion of this component is simply half the product between the first-order transition amplitudes $\mathcal{A}_{j \leftarrow i}^{(1)}$ to go (i) from the ground $1 s$ state to the resonant $n p$ state and (ii) from the resonant $n p$ state to the final state in the 


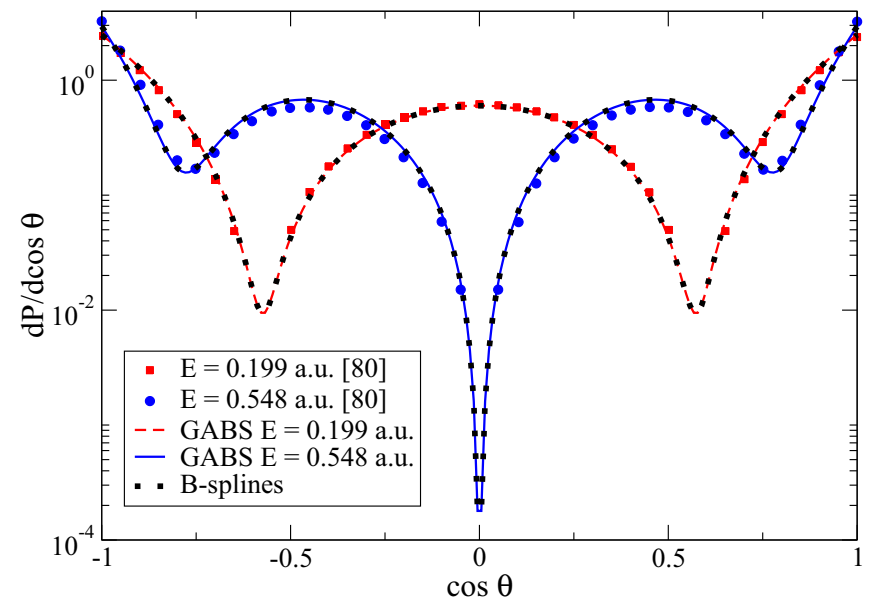

FIG. 9. (Color online) Normalized photoelectron angular distribution corresponding to the first and second ATI peaks in Fig. 8, computed here by solving the TDSE in either a $B$-spline or a GABS basis, and compared to the results obtained by Rodríguez et al. [80] in similar conditions.

continuum,

$$
\begin{aligned}
\mathcal{A}_{E \ell \leftarrow 1 s}^{(2, r)} & =-\pi \sum_{n} \mathcal{O}_{E, n p} \mathcal{O}_{n p, 1 s} \tilde{F}\left(\omega_{1 s n p}\right) \tilde{F}\left(\omega_{n p E \ell}\right) \\
& =\frac{1}{2} \sum_{n} \mathcal{A}_{E \ell \leftarrow n p}^{(1)} \mathcal{A}_{n p \leftarrow 1 s}^{(1)},
\end{aligned}
$$

where

$$
\mathcal{A}_{f i}^{(1)}=-i \sqrt{2 \pi} \tilde{F}\left(\omega_{i f}\right) \mathcal{O}_{f i} .
$$

The on-shell part of the two-photon transition amplitude is responsible for the shoulder at $E=0.225$ a.u. and for the peaks at $E \simeq 0.29$ a.u. and $E \simeq 0.32$ a.u., which correspond to the $2 p, 3 p$, and $4 p$ resonant states, respectively. Notice that the amplitudes of these peaks have the characteristic $\mathscr{F}[F]\left(E_{n p}-\right.$ $E$ ) profile of one-photon transitions; in particular, they are narrower than the nonresonant two-photon peak amplitude, which is instead proportional to $\mathscr{F}\left[F^{2}\right]\left(E_{1 s}-E\right)$, i.e., to the Fourier transform of a temporally compressed pulse. Finally, the second shoulder, starting at $E \simeq 0.25$ a.u., is the incipient signature of the wide feature with which the nonperturbative $1 s-2 p$ transition, mentioned earlier in this section, manifests in the spectrum.

In Fig. 9 we compare the angular distribution of the electrons in the region of the first and second ATI peaks with those reported by Rodríguez. Again, the agreement between the two calculations is excellent, confirming the accuracy of the observables obtained with the hybrid GABS basis. As expected, the angular distribution has a lobe structure consistent with a complex linear combination of $s+d$ amplitudes for the first ATI peak and $p+f$ amplitudes for the second ATI peak. In both cases, the two amplitudes are neither in phase nor in antiphase, hence the lack of proper nodes in the spectrum, except, in principle, for $\theta=90^{\circ}$ for the second ATI peak (even in this case, though, interference from the long-range tail of the nearby ATI peaks prevents the photoelectron distribution to attain zero).

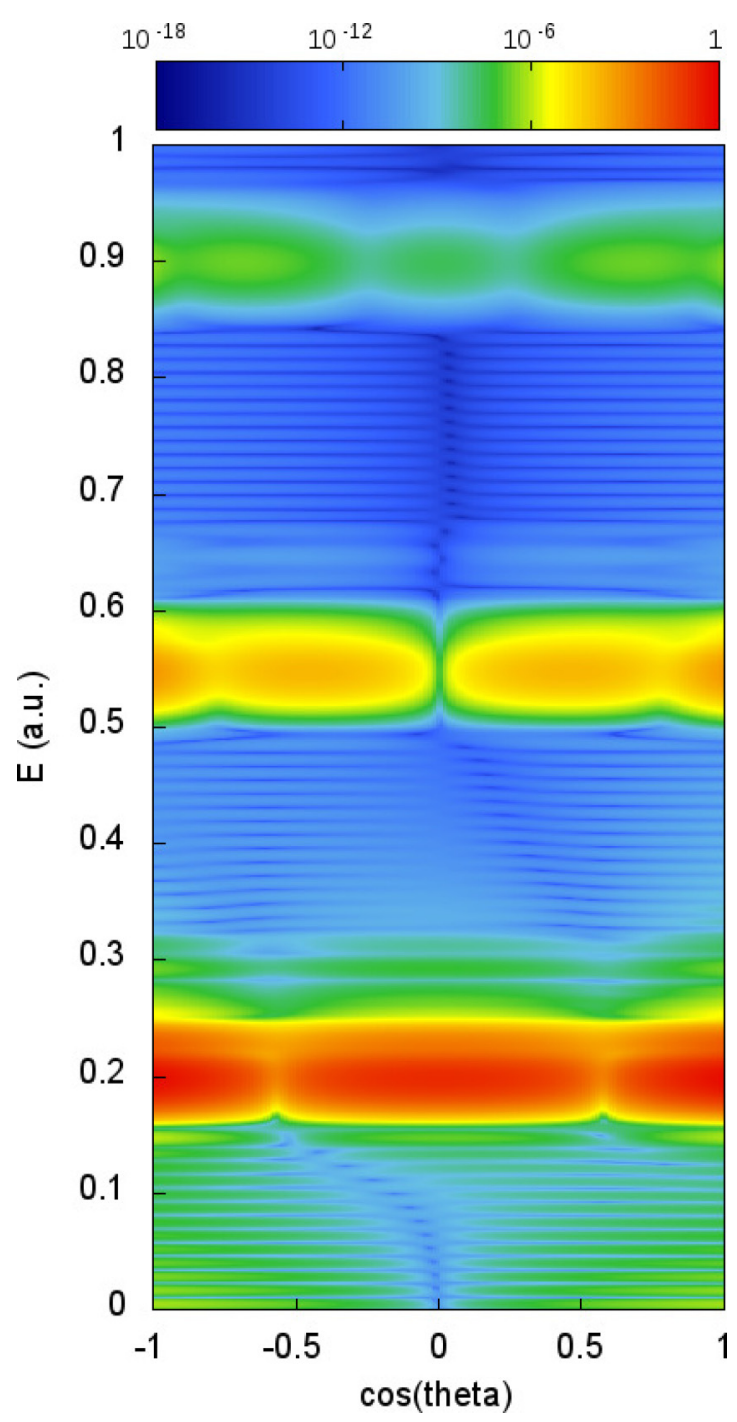

FIG. 10. (Color online) Doubly differential photoelectron distribution $d^{2} P / d E \mathrm{~d} \cos \theta$ for the process described in Fig. 8 .

To gain further insight in the angular distribution, we show in Fig. 10 the doubly differential photoelectron spectrum as a function of both the electron energy and the cosine of the ejection angle. To the best of our knowledge, such fully differential representation of the photoelectron distribution has not been reported before. While the regions corresponding to the ATI peaks visible in Fig. 10 have approximately a uniform lobe structure (three, four, and five maxima for the two-, three-, and four-photon peaks, respectively), at a closer inspection of the fully differential plot, three additional aspects emerge. First, the ATI peaks along the polarization axis $(\cos \theta= \pm 1)$ are slightly shifted to lower energies with respect to the photoelectrons ejected orthogonal to the field polarization $(\cos \theta=0)$. Second, the characteristic threemaxima angular distribution associated to the first group of peaks is slightly more pronounced for the dominant peak ( $E \simeq 0.2$ a.u.) than for the two shoulders associated with the resonant $1 s \rightarrow 2 p \rightarrow E \ell$ transition $(E \simeq 0.225$ and 0.27 a.u.). Third, the three-maxima structure in the first group of peaks is interrupted in a narrow interval around $E \simeq 0.25$ a.u., 


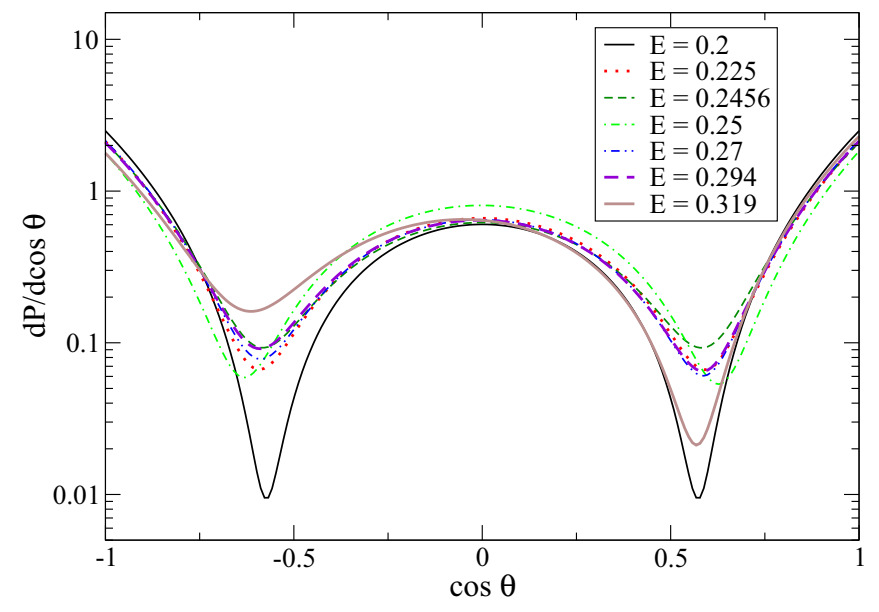

FIG. 11. (Color online) Normalized photoelectron angular distribution for several selected energies in Fig. 10. The energies 0.225, 0.294 , and 0.319 a.u. correspond to the $2 p, 3 p$, and $4 p$ resonances, respectively.

where the two dips are less pronounced. As it turns out, this is because the energy $E=0.25$ a.u. coincides with the position of the first side peak of $\mathscr{F}\left[F^{2}\right]\left(E_{1 s}-E\right)$ [compare Eq. (40)]. The presence of secondary peaks is a typical characteristics of the Fourier transform of any pulse with a compact support; the symmetric counterpart is clearly visible at $E=$ 0.15 a.u. The side peak amplitude is in antiphase with respect to that of the dominant peak. As a consequence, when interfering with the resonant component, it cancels part of the angular modulation, resulting in a more isotropic distribution. This peculiar phenomenon, which would not be visible if the external pulse had, say, a Gaussian envelope, illustrates well how special care is needed when assigning spectra generated using pulses that have a structured Fourier transform to start with.

The change of the angular distribution with energy is highlighted in Fig. 11, where we plot the normalized photoelectron angular distribution at six representative energies, $E=0.2$ a.u. (main peak of the nonresonant transition), $E=0.225$ a.u. ( $2 p$ resonant peak), $E=0.2456$ a.u. (side peak of the nonresonant transition, which corresponds to the least pronounced dips in the spectrum), $E=0.25$ a.u. (intermediate energy at which the dips are pushed towards smaller angles from the polarization axis), $E=0.27$ a.u. (second shoulder, presumably associated with the nonperturbative $2 p$ resonant transition), and $E=$ 0.294 a.u. and $E=0.319$ a.u. ( $3 p$ and $4 p$ resonant peaks, respectively). Notice that, starting from $E \simeq 0.27$ a.u., the symmetry of the photoelectron distribution is progressively broken. This feature is more dramatic for the weakest peak, the one corresponding to the resonant $4 p$ transition, for which the upward maximum $\left(\theta<90^{\circ}\right)$ is higher than the downward one $\left(\theta>90^{\circ}\right)$, while the opposite is true for the two minima. This behavior is most likely due to the interference between the weak resonant two-photon amplitude and the tail of the nonresonant three-photon amplitude, which have opposite parity [83].

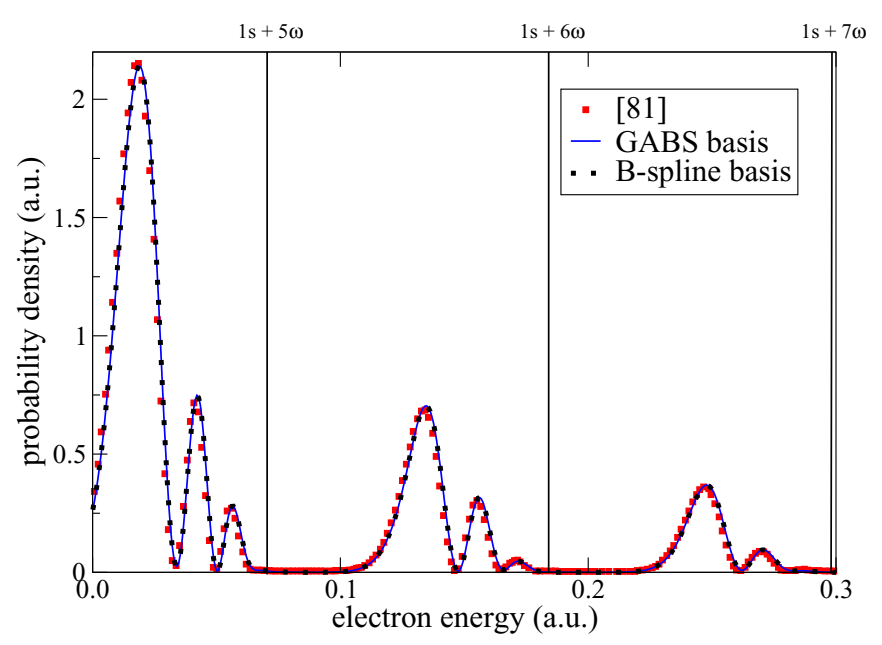

FIG. 12. (Color online) ATI photoelectron spectrum from hydrogen ground state due to a 20-cycle $\cos ^{2}$ laser pulse with $\omega=$ 0.114 a.u. and $I=0.1 \mathrm{PW} / \mathrm{cm}^{2}$. The present results, computed by solving the TDSE using either a purely $B$-spline basis (black dots) or the GABS basis (solid line), are compared with the spectrum obtained under similar conditions by Grum-Grzhimailo et al. [81] (light solid squares). See text for more details.

\section{B. Long wavelength}

As a second example, we test our simulations against the results reported by Grum-Grzhimailo et al. [81] for ionization with a 20-cycle pulse with angular frequency $\omega=$ 0.114 a.u. and peak intensity $I=10^{14} \mathrm{~W} / \mathrm{cm}^{2}$. In this case, five photons are needed to achieve ionization. As Fig. 12 shows, the agreement between the data digitized from [81] and the present calculations with GABS and the purely $B$-spline basis is again excellent. The photoelectron peaks obtained with either of the two latter bases are actually slightly shifted to lower energies with respect to those reported in [81]. Since a similar shift in the position of the peaks for high-intensity pulses was already observed in [81] as a consequence of changing the field parametrization from Eq. (33) to Eq. (34), we assume that the shift we observe here is due to a similar effect.

The most striking features of the spectrum shown in Fig. 12 are (i) the multipeak structure of each $n$-photon ATI signal and (ii) the fact that the position of the dominant peak in each group is shifted to lower energies by as much as 0.07 a.u. with respect to the nominal values $n \omega_{\mathrm{Uv}}-E_{0}$, the latter value rather approaching the upper limit of each peak subgroup. This phenomenon was first observed $25 \mathrm{y}$ ago by Bardsley et al. [84] and the mechanism at its basis was identified and described by several authors shortly thereafter [85-87]. Since essentially the same phenomenon has recently received renewed interest, in association with the use of intense ultrashort extreme ultraviolet pulses [88], in the following we briefly summarize the rationale of the underlying mechanism and contextualize our results with reference to the original [87] and latest [88] works on the subject. When a discrete state $\psi$ interacts with a moderately intense laser pulse, and as long as the laser does not resonantly couple $\psi$ to other discrete states, the energy of $\psi$ (i.e., the cycle-averaged rate of its phase modulation) changes by a quantity $\Delta E_{\psi}^{a c}$ called ac-Stark shift. For a monochromatic 
field with amplitude $E_{0}$ and angular frequency $\omega$, the ac-Stark shift $\Delta E_{1 s}^{a c}$ of the ground state of hydrogen is given, to the lowest order of perturbation theory, by the solution to the equation

$$
\Delta E_{1 s}^{a c}=\frac{E_{0}^{2}}{4} \mathcal{M}_{1 s 1 s}\left(E_{1 s}+\Delta E_{1 s}^{a c}, \omega\right)
$$

where

$$
\mathcal{M}_{i i}(E, \omega)=\left\langle i\left|z\left[G_{0}^{+}(E+\omega)+G_{0}^{+}(E-\omega)\right] z\right| i\right\rangle .
$$

The ac-Stark shift of the ground state can thus be either positive or negative depending on whether $\omega$ is, respectively, larger or smaller than the excitation energies $\omega_{\epsilon p 1 s}$ to the group of (discrete or continuum) $|\epsilon p\rangle$ states that are most strongly coupled to the ground state. The energy of the states in the continuum is affected by the ac-Stark effect as well. In this case, the ac-Stark shift is approximated reasonably well by the ponderomotive energy $\Delta E_{E \ell}^{a c} \simeq U_{p}=E_{0}^{2} / 4 \omega^{2}$, a positive quantity. Now, a careful treatment of time-dependent perturbation theory (see, e.g., Chapter 3 in [89]) shows that, in the absence of intermediate resonant states, the absorption of $n$ photons $\gamma_{\omega}$ from an initial state $|i\rangle$ to populate a final state $|f\rangle$ takes place provided that the ac-Stark shifted energies of the two dressed states differ by $n \omega, \tilde{E}_{f}=\tilde{E}_{i}+n \omega$. In the case of ionization of atomic hydrogen from the ground state, this means that the field-free energy $E$ of the final continuum state $|E \ell\rangle$ populated by the absorption of $n$ photons is approximately given by

$$
E \simeq E_{1 s}+n \omega+\Delta E_{1 s}^{a c}-U_{p} .
$$

This last equation is justified on the assumption that, when the external field switches off, the population of a dressed state follows the state adiabatically. Incidentally, such assumption is not justified if the photoelectron leaves the laser focus before the pulse is over. In this latter case, the electron is accelerated by the gradient of the ponderomotive potential that is present along the cross section of the laser beam, thus acquiring the dressing energy once and for all, instead of returning it to the field. When the light that dresses the system and induces the multiphoton transition comes in the form of a short pulse, the amplitude $E_{0}$ of the electric field associated to it changes with time, $E_{0}=E_{0}(t)$,

$$
\vec{E}(t)=\hat{\epsilon} E_{0}(t) \sin [\omega t+\phi(t)]
$$

For example, in the case of the parametrization (34) mentioned in the preceding section, the carrier is modulated by a cosinesquare envelope,

$$
E_{0}(t)=E_{0} \cos ^{2}\left(\frac{\pi t}{\tau}\right) \theta(\tau / 2-|t|) .
$$

Under these conditions, the energy $E$ of the final state that is populated by means of the absorption of $n$ photons from the hydrogen ground state changes across the pulse as well,

$$
E(t) \simeq E_{1 s}+n \omega+\frac{E_{0}^{2}(t)}{4}\left[\mathcal{M}_{1 s 1 s}\left(E_{1 s}, \omega\right)-\frac{1}{\omega^{2}}\right]
$$

(for simplicity, we disregard the dependence of $\mathcal{M}$ on the first energy entry). This phenomenon is qualitatively illustrated in Fig. 13: As the external oscillating field becomes more
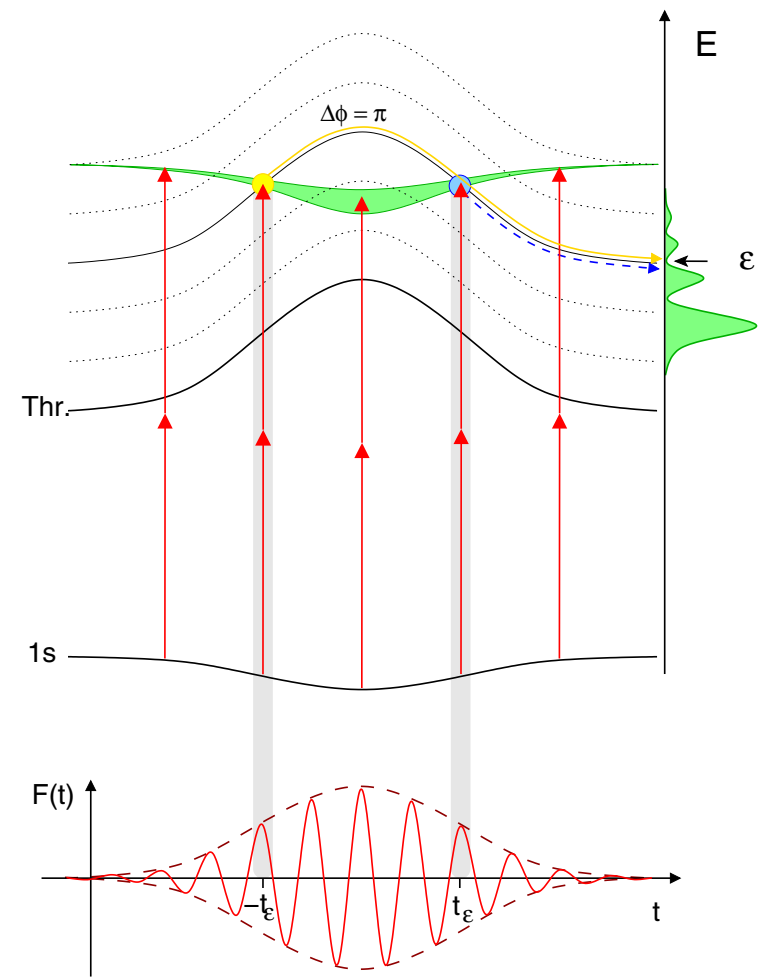

FIG. 13. (Color online) Schematic illustration of the dynamicinterference mechanism underlying the appearance of the multipeak substructure in the nonresonant multiphoton photoelectron signals when an atom is ionized with intense isolated ultrashort pulses. The external pulse induces an ac-Stark shift of the energy levels of the atom. Depending on the value of the time-dependent intensity of the laser, the pulse promotes at different times transitions to laser-dressed states that are adiabatically connected to field-free states with different energies, thus resulting in a global shift of the photoelectron signal. Two temporally separated amplitudes contribute to each final energy. The phase difference acquired by the first amplitude with respect to the second one in the intermediate time lapse can give rise to either constructive or destructive interference, thus resulting in a peak or a zero in the photoelectron spectrum, respectively. See text for more details.

and more intense, the energy of any state gets shifted by a quantity that follows the laser-intensity profile and which reaches its maximum in correspondence with the peak of the pulse envelope. As the intensity of the pulse decreases again, and eventually vanishes, the energies of the dressed states return to their original field-free value, thus traversing for a second time, in the opposite direction, all the intermediate energies. As a consequence, for a symmetric pulse centered at $t=0$, the probability amplitude to each possible final energy $\varepsilon, \mathcal{A}_{\varepsilon \leftarrow 1 s}^{(n)}$, receives two contributions: one at a time $-t_{\varepsilon}$ and another one at a time $t_{\varepsilon}$, where $E\left(t_{\varepsilon}\right)=\varepsilon$ (see Fig. 13),

$$
\mathcal{A}_{\varepsilon \leftarrow 1 s}^{(n)}=\mathcal{A}_{\varepsilon \leftarrow 1 s}^{(n)}\left(-t_{\varepsilon}\right)+\mathcal{A}_{\varepsilon \leftarrow 1 s}^{(n)}\left(t_{\varepsilon}\right) .
$$

Indeed, for $t= \pm t_{\epsilon}$, the $n$-photon transition from the ground state is resonant with the dressed state that is adiabatically connected to the field-free state with energy $\epsilon$. The two amplitudes $\mathcal{A}_{\varepsilon \leftarrow 1 s}^{(n)}\left( \pm t_{\varepsilon}\right)$ have comparable magnitude, but not the same phase. This is because, in the time interval 
$\Delta t=2 t_{\varepsilon}$ elapsed from the first transition to the second one, the amplitude of the final excited state acquires an additional phase with respect to the ground state, which is approximately given by $\Delta \phi_{\varepsilon} \simeq 2 n \omega t_{\varepsilon}$ (in this latter formula, we made the simplifying assumption that the ac-Stark shifts are small when compared with the total transition energy $n \omega)$. The two amplitudes, therefore, will interfere destructively whenever $2 n \omega t_{\varepsilon}=(2 k+1) \pi$, thus giving rise to a node in the photoelectron spectrum,

$$
P_{\varepsilon \leftarrow 1 s}^{(n)} \simeq 4\left|\mathcal{A}_{\varepsilon \leftarrow 1 s}^{(n)}\left(t_{\varepsilon}\right)\right|^{2} \cos ^{2}\left(n \omega t_{\varepsilon}\right) .
$$

The transitions taking place close to the center of the pulse, and which thus lead to the population of the final continuum state whose energy lies farther from the field-free resonant condition, all interfere constructively. As a result, in the photoelectron spectrum we observe a dominant peak at the maximally shifted final energy, accompanied by several other peaks with progressively smaller intensities and whose energy positions approach the value predicted by the field-free resonance condition $E=E_{1 s}+n \omega$.

For $\omega=0.114$ a.u., both terms in parentheses on the righthand side of (48) are negative. In our simulation, at peak intensity, the ac-Stark shifts of the ground and continuum states have the following approximate values:

$$
\Delta E_{1 s}^{a c}=-0.003 \text { a.u., } \quad U_{p}=0.055 \text { a.u. }
$$

A closer look at Fig. 12 reveals that the displacement of the largest peak with respect to the nominal position in the weak-field limit is $\simeq-0.051$ a.u. for the first groups of signals (five-photon absorption) and $\simeq-0.049$ a.u. for the second one (six-photon absorption). While both values are in qualitative agreement with the prediction $\Delta E_{\max }=\Delta E_{1 s}^{a c}-U_{p}=$ 0.058 a.u., a couple of observations are pertinent nonetheless. First, the observed shift changes from one group of peaks to the other. This is not unexpected since the photoionization cross section changes rapidly close to the threshold and the ac-Stark shift of continuum states, which is an order of magnitude larger than the ac-Stark shift of the ground state, may also change significantly in this energy range. In fact, the variation in the energy shift in the continuum is comparable to the ac-Stark shift of the ground state itself. Second, due to the short duration of the pulse, the displacement of the maximum in the spectral profile is arguably smaller than the one that would be obtained with a monochromatic laser with the same peak intensity.

The appearance of a peak substructure in the photoelectron signal for the absorption of a fixed number of photons from a short pulse has also been recently observed by Demekhin and Cederbaum [88] in the context of the ionization of the hydrogen atom by an energetic ( $\omega \simeq 2$ a.u.) intense pulse ( $I=$ $\left.5 \times 10^{15} \mathrm{~W} / \mathrm{cm}^{2}\right)$, in the region of one-photon absorption. In this case, the photoelectron signal gives rise to a group of peaks that are shifted at higher energies with respect to the nominal position of the peak in the weak-field limit, rather than at smaller energies as in the case discussed in this paper. This is because, for $\omega_{\mathrm{Uv}} \simeq 2$ a.u., the ac-Stark shift of the ground state is (i) positive and (ii) larger than the ponderomotive shift experienced by the final continuum states.

In Fig. 14, we report the fully differential photoelectron distribution corresponding to the spectrum shown in Fig. 12,

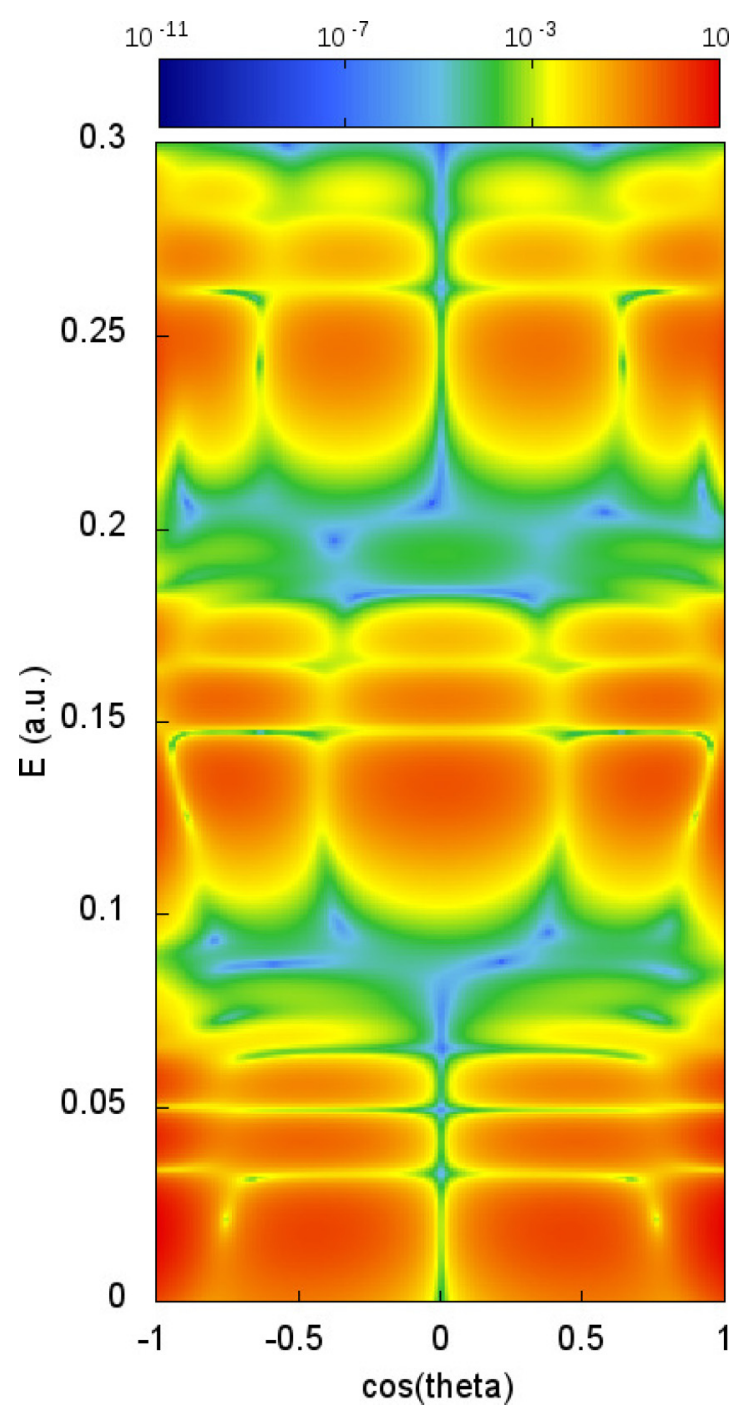

FIG. 14. (Color online) Doubly differential photoelectron distribution $d^{2} P / d E \mathrm{~d} \cos \theta$ for the process described in Fig. 12 .

computed with the GABS basis. When compared with the analogous spectrum in Fig. 10, obtained with a higher laser frequency and lower pulse intensity, it is apparent that the structure is more complicated. Here the correspondence between the number of dips in the angular distribution and the number of absorbed photons is not visible anymore. Indeed, the first group of peaks, comprised between the threshold and $E \simeq 0.08$ a.u., which results from the absorption of five photons, counts at most four local maxima. Furthermore, the downward energy shift of the signal for the photoelectrons ejected along the polarization axis with respect to those ejected perpendicularly to it, which was barely visible in Fig. 10, is here much more pronounced. This effect, which is clearly visible for the two absolute maxima in the group of peaks comprised between $E \simeq 0.09$ a.u. and $E \simeq 0.2$ a.u. (the first group of peaks is truncated by the threshold opening) constitutes an additional aspect of the dynamical-shift effect that cannot be detected in a two-dimensional plot like Fig. 12. The spectral lines that are most dramatically distorted by this effect are the weakest ones within each group, i.e., at 
$E \simeq 0.07$ a.u. and $E \simeq 0.19$ a.u., which are also the ones closer to the nominal weak-field positions of the ATI peaks. A final peculiar aspect that we would like to highlight in Fig. 14 is the appearance of isolated narrow dips in the transition probability, e.g., for $(E, \cos \theta) \simeq(0.03$ a.u., \pm 0.7$),(0.02$ a.u., \pm 0.75$)$, ( 0.125 a.u., \pm 0.9$)$. This phenomenon is associated with the fact that the transition amplitude beneath the photoelectron distribution is complex, rather than real. As a result, the loci of zeros in the $(E, \cos \theta)$ domain of its real and imaginary components are curves that generally intersect at isolated points only. Symmetry nodes, like those at $\theta=90^{\circ}$ for the absorption of an odd number of photons, are a notable exception.

\section{CONCLUSIONS}

We have introduced a hybrid GABS for the description of the continuum that permits to achieve high electron energies while, at the same time, preserving a purely Gaussian representation of the electronic wave function in a radial region large enough to encompass whole polyelectronic atoms as well as small molecules. We have demonstrated that the hybrid GABS basis can be used to compute with high accuracy all the observables associated with the interaction of a single-active electron atom with external light pulses in a wide energy range. In particular, we have reproduced with the GABS basis the energies and transition amplitudes of the hydrogen atom as well as the fully differential photoelectron distributions that result from the interaction of the atom with strong ultra-short external laser pulses. All the results are in excellent agreement with those obtained numerically from well-established $B$ spline bases, with data taken from the literature and with analytical predictions, when available.

In the analysis of the results from the solution of the TDSE for the hydrogen atom exposed to external pulses, we have presented and discussed new fully differential photoelectron distributions of the hydrogen ATI spectra obtained in the conditions reported in two recent works $[80,81]$. In particular, we contextualize the low-energy multipeak structure of the ATI spectrum of hydrogen reported by Grum-Grzhimailo and co-workers [81] and the dynamic-interference mechanism highlighted recently by Demekhin and Cederbaum [88] within the theoretical framework outlined long ago by Cormier and Lambropoulos [87].

In conclusion, the GABS basis has the benefit of the most flexible numerical basis for the description of the continuum while at the same time being expressed in terms of standard Gaussian functions in a large inner radial region. These features make GABS a very attractive candidate for the extension of existing quantum-chemistry packages to the calculation of molecular continuum properties.

\section{ACKNOWLEDGMENTS}

All calculations were performed at Mare Nostrum BSC and CCC-UAM. Work supported by the European Research Council under the European Union's Seventh Framework Programme (FP7/2007-2013)/ERC Grant Agreement No. 290853, European COST Action No. CM1204 XLIC, and MICINN Project No. FIS2010-15127.

\section{APPENDIX A: DIPOLE TRANSITION GAUGES}

In this section we explore in detail how dipole transition matrix elements evaluated between eigenstates of either the unconstrained or the confined hydrogen Hamiltonian (physical and confined eigenstates, respectively, in the nomenclature of Sec. II), computed in different gauges, are related to each other. In the case of the hydrogen Hamiltonian,

$$
H=\frac{1}{2} p^{2}-\frac{Z}{r},
$$

the following operator identities hold:

$$
\begin{gathered}
{\left[H, r_{1 \mu}\right]=\frac{1}{2}\left[p^{2}, r_{1 \mu}\right]=-i p_{1 \mu},} \\
{\left[H, p_{1 \mu}\right]=-Z\left[\frac{1}{r}, p_{1 \mu}\right]=i Z \frac{r_{1 \mu}}{r^{3}} .}
\end{gathered}
$$

As a consequence, if we indicate with $\psi_{E \ell m}$ and $\psi_{E^{\prime} \ell^{\prime} m^{\prime}}$ two generic eigenstates of $H$ with eigenvalues $E$ and $E^{\prime}$, respectively, we can easily derive the following relations between the off-shell $\left(E \neq E^{\prime}\right)$ reduced transition matrix elements:

$$
\begin{aligned}
i\left(E-E^{\prime}\right)\left\langle\psi_{E \ell}\left\|\mathcal{O}_{1}^{(l)}\right\| \psi_{E^{\prime} \ell^{\prime}}\right\rangle & =\left\langle\psi_{E \ell}\left\|\mathcal{O}_{1}^{(v)}\right\| \psi_{E^{\prime} \ell^{\prime}}\right\rangle \\
-i\left(E-E^{\prime}\right)\left\langle\psi_{E \ell}\left\|\mathcal{O}_{1}^{(v)}\right\| \psi_{E^{\prime} \ell^{\prime}}\right\rangle & =\left\langle\psi_{E \ell}\left\|\mathcal{O}_{1}^{(a)}\right\| \psi_{E^{\prime} \ell^{\prime}}\right\rangle .
\end{aligned}
$$

These equations have two uses. First, the same off-shell transition matrix element (say, the one in velocity gauge) can be computed in three different ways. Since the kernels of the three transition operators [Eq. (19)] weighs differently the wave function at short and long range, one can choose the form that weighs more the region where the wave function is known to be computed with better accuracy. Second, Eqs. (A4) and (A5) only hold when evaluated using exact eigenstates of the Hamiltonian. The discrepancy between the numerical realization of the right-hand side and the left-hand side in (A4) and (A5), therefore, is a measure of the accuracy of the numerical eigenstates.

Three further remarks are in order about the relations (A4) and (A5). First, as it has already been pointed out, these relations permit to convert between each other only off-shell matrix elements. In the on-shell case, where the two eigenstates are necessarily either both bound or both in the continuum, the relations provide only partial information. For example, they say that the transition matrix elements in velocity and acceleration gauge between degenerate bound states is exactly zero, but they do not say anything about the finite value of the transition matrix elements in length gauge between degenerate states (e.g., between the $2 s$ and the $2 p$ states). Second, when evaluated between two continuum generalized eigenstates and expressed in terms of a radial integral, the transition matrix elements in length and velocity gauge must be regularized with the inclusion of an exponential extinction factor for the integral to converge in the first place. The transition matrix element in acceleration gauge, on the other hand, is a continuous function. As a consequence, Eqs. (A5) is to be seen as a relation between distributions. The general expression of the velocity-gauge transition matrix element in terms of the acceleration gauge is 
thus [90-92]

$$
\begin{aligned}
\left\langle\psi_{E \ell-1}\left\|\mathcal{O}_{1}^{(v)}\right\| \psi_{E^{\prime} \ell}\right\rangle= & i P \frac{\left\langle\psi_{E \ell-1}\left\|\mathcal{O}_{1}^{(a)}\right\| \psi_{E^{\prime} \ell}\right\rangle}{E-E^{\prime}} \\
& +\mathcal{F}_{\ell E} \delta\left(E-E^{\prime}\right),
\end{aligned}
$$

where $P$ indicates the principal part and $\mathcal{F}_{\ell E}$ is a function that depends on the asymptotic behavior of the radial part of two functions and must be determined separately. In Appendix B we derive the explicit expression for $\mathcal{F}_{\ell E}$ for the hydrogen atom as well as in the presence of a short-range potential. Notice that the on-shell continuum-continuum integral in length gauge cannot be regularized, so Eq. (A4) does not have an extension similar to what Eq. (A6) is for (A5). Third, the use of the term "gauge" for the acceleration operator is admittedly an abuse of language. Indeed, while the velocity and length gauges are related to each other by a unitary transformation (Göppert-Mayer's; see [93]), this is not the case for the acceleration operator. In the present context, the latter should thus be regarded simply as an alternative way to estimate the transition matrix element in velocity gauge.

When computing the transition matrix elements between box eigenstates, the considerations above need to be modified. Indeed, in this case the Hamiltonian can be regarded as the limit of the hydrogen Hamiltonian plus a step potential at $r=R_{\text {box }}$, with height $V$, for $V \rightarrow \infty$,

$$
H(V)=\frac{p^{2}}{2}-\frac{Z}{r}+V \theta\left(r-R_{\mathrm{box}}\right),
$$

where we explicitly indicated the parametric dependence of $H$ on $V$. As long as we are interested in transition matrix elements between eigenstates with eigenvalues smaller than $V$, the relation (A4) between velocity and length transition matrix elements continues to apply as it does between bound states of the unconstrained hydrogen Hamiltonian. In particular, the equivalence (A4) holds in the limit $V \rightarrow \infty$, i.e., between box eigenstates. This is because the commutator (A2) is unaltered by the presence of an additional multiplicative term in the Hamiltonian in coordinate representation. Notice, however, that while the positive-energy box eigenstates can be normalized so to coincide, within the box, with the real scattering states with the same energy, the transition matrix elements between box eigenstates (i.e., where the radial integral is truncated at $r=R_{\text {box }}$, where all the box eigenstates vanish) does not coincide with the one evaluated between scattering states; the contribution to the (regularized) transition integral from the radial domain $\left[R_{\mathrm{box}}, \infty\right)$ is finite and non-negligible [76]. The picture takes on a different perspective when the acceleration gauge is considered. In this case, the right-hand side of (A3) changes,

$$
\begin{aligned}
{\left[H, p_{1 \mu}\right] } & =-Z\left[\frac{1}{r}, p_{1 \mu}\right]+V\left[\theta\left(r-R_{\mathrm{box}}\right), p_{1 \mu}\right] \\
& =i Z \frac{r_{1 \mu}}{r^{3}}+i V \frac{r_{1 \mu}}{r} \delta\left(r-R_{\mathrm{box}}\right) .
\end{aligned}
$$

This latter equivalence between the left-hand side and the right-hand side still holds for arbitrary values of $V$, but the limit for $V \rightarrow \infty$ of the new term on the right-hand side evaluated between eigenstates of the Hamiltonian that comprises the step potential does not vanish and must be taken into account. If we indicate with $\psi_{E \ell m}(r ; V)=r^{-1} u_{\ell E}(r ; V) Y_{\ell m}(\hat{r})$ a generic eigenstate of the Hamiltonian that includes the step potential, where the parametric dependence on $V$ has explicitly been indicated and we assume $V \gg E$, then we can immediately write, for $r \geqslant R_{\text {box }}$,

$$
\begin{gathered}
u_{\ell E}(r ; V) \simeq N(E, V) e^{-\kappa\left(r-R_{\mathrm{box}}\right)}, \\
u_{\ell E}^{\prime}(r ; V) \simeq-\kappa N(E, V) e^{-\kappa\left(r-R_{\mathrm{box}}\right)},
\end{gathered}
$$

where $\kappa=\sqrt{2(V-E)}$ and $N(E, V)$ is a normalization constant. In the limit $V \rightarrow \infty$, if the wave function is to remain normalized, the wave function at the box boundary must converge to zero while its derivative must converge to a finite value. Therefore, we can choose the normalization so that the wave function derivative coincides with its asymptotic value for any $V \gg E$,

$$
\begin{aligned}
u_{\ell E}(r ; V) & \simeq-\frac{u_{\ell E}^{\prime}\left(R_{\mathrm{box}}, \infty\right)}{\sqrt{2(V-E)}} e^{-\kappa\left(r-R_{\mathrm{box}}\right)} \\
& \simeq-\frac{u_{\ell E}^{\prime}\left(R_{\mathrm{box}}, \infty\right)}{\sqrt{2 V}} e^{-\kappa\left(r-R_{\mathrm{box}}\right)}, \\
u_{\ell E}^{\prime}(r ; V) & \simeq u_{\ell E}^{\prime}\left(R_{\mathrm{box}}, \infty\right) e^{-\kappa\left(r-R_{\mathrm{box}}\right)} .
\end{aligned}
$$

Now we can compute the limit of the matrix element of the second term on the right-hand side of Eq. (A8) (we assume radial wave functions to be real):

$$
\begin{aligned}
& \left\langle\psi_{E \ell m}\left|i V \frac{r_{1 \mu}}{r} \delta\left(r-R_{\mathrm{box}}\right)\right| \psi_{E^{\prime} \ell^{\prime} m^{\prime}}\right\rangle \\
& =i\left\langle Y_{\ell m}\left|\frac{r_{1 \mu}}{r}\right| Y_{\ell^{\prime} m^{\prime}}\right\rangle V u_{E \ell}\left(R_{\mathrm{box}} ; V\right) u_{E^{\prime} \ell^{\prime}}\left(R_{\mathrm{box}} ; V\right) \\
& \simeq \frac{C_{\ell^{\prime} m^{\prime}, 1 \mu}^{\ell m}}{\sqrt{2 \ell+1}} \frac{i}{2} \sqrt{2 \ell^{\prime}+1} C_{\ell^{\prime} 0,10}^{\ell 0} \\
& \quad \times u_{\ell E}^{\prime}\left(R_{\mathrm{box}} ; \infty\right) u_{\ell^{\prime} E^{\prime}}^{\prime}\left(R_{\mathrm{box}} ; \infty\right) .
\end{aligned}
$$

The increase of the barrier height, therefore, exactly compensates the decrease of the wave function at the boundary. In the limit $V \rightarrow \infty$, the last approximate equality becomes an identity. In conclusion, even if the transition matrix elements in velocity and acceleration gauges, defined as in (19), do not satisfy the relation (A5) when computed between box eigenstates unless the radial derivative of at least one of them is vanishingly small at the box boundary, the conversion from one gauge to the other can still be performed provided that the additional term (A14), which we call CBC, is taken into account,

$$
\begin{aligned}
(E- & \left.E^{\prime}\right)\left\langle\psi_{E \ell}\left\|\mathcal{O}_{1}^{(v)}\right\| \psi_{E^{\prime} \ell^{\prime}}\right\rangle_{r \in\left[0, R_{\mathrm{box}}\right]} \\
= & i\left\langle\psi_{E \ell}\left\|\mathcal{O}_{1}^{(a)}\right\| \psi_{E^{\prime} \ell^{\prime}}\right\rangle_{r \in\left[0, R_{\mathrm{box}}\right]} \\
& +\frac{i}{2} \sqrt{2 \ell^{\prime}+1} C_{\ell^{\prime} 0,10}^{\ell 0} u_{E \ell}^{\prime}\left(R_{\mathrm{box}}\right) u_{E^{\prime} \ell^{\prime}}^{\prime}\left(R_{\mathrm{box}}\right) .
\end{aligned}
$$

Finally, if the box size is large enough, thanks to the $r^{-2}$ behavior of the acceleration kernel, the acceleration matrix element between any two box eigenstates does converge to those between the real eigenstates of the unconstrained Hamiltonian,

$$
\lim _{R_{\mathrm{box}} \rightarrow \infty}\left\langle\psi_{E \ell}\left\|\mathcal{O}_{1}^{(a)}\right\| \psi_{E^{\prime} \ell^{\prime}}\right\rangle_{r \in\left[R_{\mathrm{box}}, \infty\right)}=0
$$

As a consequence, the contribution to the regularized velocity transition integral from the $\left[R_{\mathrm{box}}, \infty\right)$ radial domain, evaluated 
between the analytic extensions of the box eigenfunctions, can be obtained, for large values of $R_{\mathrm{box}}$, from the correction in (A14),

$$
\begin{aligned}
& \left(E-E^{\prime}\right)\left\langle\psi_{E \ell}\left\|\mathcal{O}_{1}^{(v)}\right\| \psi_{E^{\prime} \ell^{\prime}}\right\rangle_{r \in\left[R_{\mathrm{box}}, \infty\right)} \\
& =-\frac{i}{2} \sqrt{2 \ell^{\prime}+1} C_{\ell^{\prime} 0,10}^{\ell 0} u_{E \ell}^{\prime}\left(R_{\mathrm{box}}\right) u_{E^{\prime} \ell^{\prime}}^{\prime}\left(R_{\mathrm{box}}\right) .
\end{aligned}
$$

This last equation has two distinctive features that set it apart from similar corrections available in the literature. First, it is a closed expression which, together with the transition integral truncated to $R_{\text {box }}$, provides a transition matrix element which is as accurate as the one that can be obtained in the same box using the acceleration gauge instead. This means that Eq. (A17) offers the opportunity to extend to continuum-continuum transitions the stringent gauge-invariance test employed for transitions from or to bound states and thus provides an independent way to assess the accuracy of the discretized continuum functions computed numerically. Second, Eq. (A17) is only applicable to the fixed energies, for both $\ell$ and $\ell^{\prime}$ angular momenta, that result from imposing box boundary conditions. In Appendix D we derive an approximated expression that does not have this restriction.

\section{APPENDIX B: SINGULAR PART OF THE DIPOLE MATRIX ELEMENT IN VELOCITY GAUGE BETWEEN CONTINUUM STATES}

As observed in Appendix A, the relation between continuum-continuum dipole transition matrix elements in velocity and acceleration gauges includes a singular on-shell contribution, according to the complete gauge relation (A6). To evaluate the factor $\mathcal{F}_{\ell E}$ in (A6), we start from the well-known formula [73]

$$
\begin{aligned}
& \left\langle\psi_{E \ell-1}\left\|\nabla_{1}\right\| \psi_{E^{\prime}, \ell}\right\rangle \\
& =-\sqrt{\ell} \int_{0}^{\infty} d r u_{\ell-1 E}^{*}(r)\left(\frac{d}{d r}+\frac{\ell+1}{r}\right) u_{\ell, E^{\prime}}(r),
\end{aligned}
$$

where $u_{\ell E}(r)$ is a reduced radial function. Since we are interested in the singular part of the integral, we can disregard the contribution to the integral from any finite interval $[0, R]$. As a consequence, we can replace $u_{\ell E}(r)$ with its asymptotic expression [72],

$$
\begin{gathered}
u_{\ell E}(r) \simeq \sqrt{\frac{2}{\pi k}} \sin \theta_{\ell k}(r), \\
\theta_{\ell k}(r)=k r-\gamma \ln 2 k r-\frac{\ell \pi}{2}+\sigma_{\ell}+\delta_{\ell},
\end{gathered}
$$

and disregard entirely the term that comes from the operator $r^{-1}$. As usual, in Eq. (B2), $k=\sqrt{2 E}, \gamma=-Z / k$, $\sigma_{\ell}=\arg \Gamma(\ell+1+i \gamma)$ is the Coulomb phase, and $\delta_{\ell}$ is the additional phase due to a possible short-range potential (for hydrogen, $Z=1$ and $\delta_{\ell}=0$ ). On this basis, the factor $\mathcal{F}_{\ell E}$ can be computed as

$$
\begin{aligned}
\mathcal{F}_{\ell E} & =\int_{E-\epsilon}^{E+\epsilon} d E^{\prime}\left\langle\psi_{E \ell-1}\left\|\mathcal{O}_{1}^{(v)}\right\| \psi_{E^{\prime}, \ell}\right\rangle \\
& =\frac{2 i \sqrt{\ell}}{\pi k} \int_{E-\epsilon}^{E+\epsilon} d E^{\prime} \int_{R}^{\infty} d r \sin \theta_{\ell-1 k}(r) \frac{d}{d r} \sin \theta_{\ell k^{\prime}}(r),
\end{aligned}
$$

in the limit of $\epsilon \rightarrow 0^{+}$and $R \rightarrow \infty$. What matters in the evaluation of the singular part of the radial integral in (B5) is that the oscillations of degenerate initial and final states are in a fixed phase relation across the whole radial range. This is true independently of the presence of the asymptotic logarithmic term in (B2). Indeed, it is sufficient to perform the change of variable $r \mapsto r^{\prime}(r)=r-\gamma / k \ln 2 k r, d r^{\prime}=$ $\left[1-\gamma /\left(2 k^{2} r\right)\right] d r$ to realize that the logarithmic term can be safely ignored on the same ground that the term $(\ell+1) r^{-1}$ in the transition operator was. Therefore, the radial integral on the right-hand side in the last equation is equivalent to the expression

$$
\begin{aligned}
& k \int_{0}^{\infty} d r \sin \left(k r+\sigma_{\ell-1}+\delta_{\ell-1}-(\ell-1) \pi / 2\right) \\
& \quad \times \cos \left(k^{\prime} r+\sigma_{\ell}+\delta_{\ell}-\ell \pi / 2\right)
\end{aligned}
$$

which does not depend on $R$ [innermost limit in (B5)]. It takes only few passages to show that the singular component of this integral is

$$
\frac{k \pi}{2} \delta\left(k-k^{\prime}\right) \cos \left(\sigma_{\ell}+\delta_{\ell}-\sigma_{\ell-1}-\delta_{\ell-1}\right) .
$$

In conclusion,

$$
\mathcal{F}_{\ell E}=i \sqrt{\ell} k \cos \left(\sigma_{\ell}+\delta_{\ell}-\sigma_{\ell-1}-\delta_{\ell-1}\right) .
$$

In the case of the hydrogen atom, $\delta_{\ell}=0 \forall \ell$, and we can write

$$
\begin{aligned}
\mathcal{F}_{\ell E} & =i \frac{\ell^{1 / 2} k}{2}\left[\frac{|\Gamma(\ell+i \gamma)|}{\Gamma(\ell+i \gamma)} \frac{\Gamma(\ell+1+i \gamma)}{|\Gamma(\ell+1+i \gamma)|}+\text { c.c. }\right] \\
& =\frac{2 i \ell^{3 / 2} E}{\sqrt{2 \ell^{2} E+1}},
\end{aligned}
$$

where in the last passage we made use of the relation $\Gamma(s)=$ $(s-1) \Gamma(s-1)$.

\section{APPENDIX C: ANALYTICAL DIPOLE MATRIX ELEMENTS IN HYDROGEN}

The equation for the reduced radial Coulomb problem of an electron in interaction with a point charge $Z$ is

$$
\begin{gathered}
\psi_{k \ell m}(\vec{r})=\frac{u_{\ell k}(r)}{r} Y_{\ell m}(\hat{r}), \\
{\left[\frac{d^{2}}{d r^{2}}+k^{2}-\frac{\ell(\ell+1)}{r^{2}}+\frac{2 Z}{r}\right] u_{\ell k}(r)=0 .}
\end{gathered}
$$

For negative energies, we can set $\kappa=i \sqrt{2|E|}$ (i.e., we choose the determination on the physical sheet of $\sqrt{2 E}$ ) and obtain

$$
\left[\frac{d^{2}}{d \rho^{2}}+\frac{2 \gamma}{\rho}-\frac{\ell(\ell+1)}{\rho^{2}}-1\right] y_{\ell \kappa}(\rho)=0,
$$

where we have defined $\gamma=Z /|\kappa|, \rho=|\kappa| r$, and $y_{\ell k}(\rho)=$ $u_{\ell k}(r)$. The solution to the latter equation that is regular at $r \rightarrow \infty$ can be expressed as

$$
y_{\ell k}(\rho) \propto e^{-\rho}(2 \rho)^{\ell+1} U(\ell+1-\gamma, 2 \ell+2,2 \rho),
$$

where $U(a, b, z)$ is the confluent hypergeometric function of the second kind [70],

$$
\begin{aligned}
U(a, b, z)= & \frac{\Gamma(1-b)}{\Gamma(a-b+1)}{ }_{1} F_{1}(a ; b ; z) \\
& +\frac{\Gamma(b-1)}{\Gamma(a)} z^{1-b}{ }_{1} F_{1}(a-b+1 ; 2-b ; z),
\end{aligned}
$$


${ }_{1} F_{1}(\alpha ; \beta ; z)$ being the ordinary confluent hypergeometric function

$$
{ }_{1} F_{1}(\alpha ; \beta ; z)=\sum_{j=0}^{\infty} \frac{(\alpha)_{j} z^{j}}{(\beta)_{j} j !}, \quad(\alpha)_{0}=1, \quad(\alpha)_{n}=\prod_{j=0}^{n-1}(\alpha+j) .
$$

Even in the presence of short-range potentials, (C4) expresses the general asymptotic form that must be fulfilled by the bound states of the system. To determine the acceptable bound-state energies, the logarithmic derivative of (C4) must match, at a radius $R$ beyond which the short-range potential is negligible, that of the degenerate solution that is regular at the origin. This is how we obtained Eq. (17) in Sec. II B. The function $U(\ell+$ $1-\gamma, 2 \ell+2,2 \rho)$ is irregular at the origin except for integer values of $\gamma, \gamma=n>\ell$. In this latter case, $U$ is proportional to a Laguerre polynomial (13.6.27 in [70]),

$$
U(-k, 2 \ell+2, z)=(-1)^{k} k ! L_{k}^{(2 \ell+1)}(z), \quad k \in \mathbb{N}_{0},
$$

and (C4) thus identifies with an admissible bound state of the hydrogenlike system. The reduced radial component $u_{n \ell}$ of the normalized hydrogen bound states $\psi_{n \ell m}$, with energy $E_{n}=-1 / 2 n^{2}$, angular momentum $\ell$, and projection $m$, have the expression [72]

$$
u_{n \ell}(r)=N_{n \ell} \rho^{\ell+1} e^{-i \rho}{ }_{1} F_{1}(\ell+1-i \eta ; 2 \ell+2 ; 2 i \rho),
$$

where, for reasons that will be clear in a moment, we introduced the new variables $\kappa=i / n, \eta=-1 / \kappa,(\eta=i n)$, and $\rho=\kappa r$, while the normalization factor $N_{n \ell}$ is given by

$$
N_{n \ell}=\frac{2^{\ell+1} n^{-1}}{i^{\ell+1}(2 \ell+1) !} \sqrt{\frac{(n+\ell) !}{(n-\ell-1) !}} .
$$

In the case of the continuum states, the reduced radial part of the wave function $\psi_{E \ell m}$, normalized as $\left\langle\psi_{E \ell m} \mid \psi_{E^{\prime} \ell m}\right\rangle=$ $\delta\left(E-E^{\prime}\right)$, is [72]

$$
u_{\ell E}(r)=C_{\ell E} \rho^{\ell+1} e^{-i \rho}{ }_{1} F_{1}(\ell+1-i \gamma ; 2 \ell+2 ; 2 i \rho),
$$

where

$$
\begin{gathered}
C_{\ell E}=\sqrt{\frac{2}{\pi k}} C_{\ell}(\gamma), \\
C_{\ell}(\gamma)=2^{\ell} e^{-\frac{\pi}{2} \gamma} \frac{|\Gamma(\ell+1-i \gamma)|}{(2 \ell+1) !},
\end{gathered}
$$

$k=\sqrt{2 E}, \gamma=-1 / k, \rho=k r$, and $C_{\ell}(\gamma)$ is the Gamow factor. Apart for the normalization factor, therefore, the expressions for the continuum and bound eigenstates are simply the analytical continuation to the real and imaginary axis of a function of the generalized radial momentum $k$,

$$
y_{\ell k}(r)=\rho^{\ell+1} e^{-i \rho}{ }_{1} F_{1}(\ell+1-i \gamma ; 2 \ell+2 ; 2 i \rho) .
$$

In fact, the normalization factor for the bound states can be cast in the suggestive form

$$
\begin{aligned}
N_{n \ell}= & n^{-3 / 2} \sqrt{1-e^{2 \pi \eta}}(-1)^{\ell+1} 2^{\ell} \sqrt{\frac{2}{\pi \kappa}} e^{-\frac{\pi}{2} \eta} \\
& \times \frac{\sqrt{\Gamma(\ell+1-i \eta) \Gamma(\ell+1+i \eta)}}{(2 \ell+1) !},
\end{aligned}
$$

where the proper analytical continuation of the normalization factor for the continuum states has been factored out. (Notice that, in this latter formulation, $N_{n \ell}$ is defined for integer values of $n$ only in terms of the limit for $\eta \rightarrow i n$, thanks to the compensation of two divergences). This means that the normalized bound eigenstates of hydrogen can be obtained as the analytical continuation of the continuum eigenstates as

$$
u_{n \ell}(r)=i^{2 \ell+1} n^{-3 / 2} \lim _{\gamma \rightarrow i n} \sqrt{1-e^{2 \pi \gamma}} u_{\ell E}(r) .
$$

In the following, we summarize the analytical expressions for the off-shell reduced dipole transition matrix elements, in length gauge, between arbitrary hydrogen eigenstates,

$$
\left\langle\psi_{a \ell^{\prime}}\left\|r_{1}\right\| \psi_{b \ell}\right\rangle=\prod_{\ell} C_{\ell 0,10}^{\ell^{\prime} 0} \int_{0}^{\infty} u_{\ell^{\prime} a}^{*} r u_{\ell b} d r
$$

where $\prod_{\ell_{1} \ell_{2} \ldots}=\sqrt{\left(2 \ell_{1}+1\right)\left(2 \ell_{2}+1\right) \cdots}$ and $C_{\ell_{1} m_{1}, \ell_{2} m_{2}}^{\ell_{3} m_{3}}$ is a Clebsch-Gordan coefficient. The expression for the corresponding quantities in the other two gauges can be readily obtained using relations (A4) and (A5). The on-shell case has already been thoroughly discussed in the previous two appendixes. We focus the attention on the radial integral common to all these cases,

$$
\mathcal{R}_{\ell^{\prime} a, \ell b}=\int_{0}^{\infty} y_{\ell^{\prime} a}^{*}(r) r y_{\ell b}(r) d r,
$$

where $a$ and $b$ are complex numbers in the first quadrant. The values pertinent to the continuum and bound indexes are obtained by taking the limit to the positive real and positive imaginary axes, respectively,

$$
\begin{aligned}
\mathcal{R}_{\ell^{\prime} \kappa^{\prime}, \ell \kappa}= & \left(\kappa^{\prime}\right)^{\ell^{\prime}+1} \kappa^{\ell+1} \int_{0}^{\infty} d r r^{\ell+\ell^{\prime}+3} e^{-i\left(\kappa+\kappa^{\prime}\right) r} \\
& \times{ }_{1} F_{1}\left(\ell^{\prime}+1-i \eta^{\prime} ; 2 \ell^{\prime}+2 ; 2 i \kappa^{\prime} r\right) \\
& \times{ }_{1} F_{1}(\ell+1-i \eta ; 2 \ell+2 ; 2 i \kappa r) .
\end{aligned}
$$

The integral in Eq. (C18) is of the form [94]

$$
\begin{aligned}
J_{\beta}^{s, p}\left(\alpha, \alpha^{\prime}\right)= & \int_{0}^{\infty} d r e^{-\left(h+h^{\prime}\right) \frac{r}{2}} r^{\beta-1+s} \\
& \times{ }_{1} F_{1}(\alpha ; \beta ; h r){ }_{1} F_{1}\left(\alpha^{\prime} ; \beta-p ; h^{\prime} r\right),
\end{aligned}
$$

which can be solved by applying repeatedly the well-known recurrence relations [94]

$$
J_{\beta}^{s, p}\left(\alpha, \alpha^{\prime}\right)=\frac{(\beta-1)}{h}\left[J_{\beta-1}^{s, p-1}\left(\alpha, \alpha^{\prime}\right)-J_{\beta-1}^{s, p-1}\left(\alpha-1, \alpha^{\prime}\right)\right],
$$

$$
\begin{aligned}
J_{\beta}^{s+1,0}\left(\alpha, \alpha^{\prime}\right)= & \frac{4}{h^{2}-h^{\prime 2}}\left\{2 \alpha^{\prime} s J_{\beta}^{s-1,0}\left(\alpha, \alpha^{\prime}+1\right)\right. \\
& +\left[\frac{1}{2} \beta\left(h-h^{\prime}\right)-h \alpha+h^{\prime}\left(\alpha^{\prime}-s\right)\right] J_{\beta}^{s, 0}\left(\alpha, \alpha^{\prime}\right) \\
& \left.+s\left(\beta-1+s-2 \alpha^{\prime}\right) J_{\beta}^{s-1,0}\left(\alpha, \alpha^{\prime}\right)\right\}, \quad(\mathrm{C} 21)
\end{aligned}
$$


until the initial expression is entirely given in terms of $J_{\beta}^{0,0}$ integrals, and finally computing

$$
\begin{aligned}
J_{\beta}^{0,0}\left(\alpha, \alpha^{\prime}\right)= & 2^{\beta} \Gamma(\beta)\left(h+h^{\prime}\right)^{\alpha+\alpha^{\prime}-\beta}\left(h^{\prime}-h\right)^{-\alpha} \\
& \times\left(h-h^{\prime}\right)^{-\alpha^{\prime}}{ }_{2} F_{1}\left[\alpha, \alpha^{\prime} ; \beta ;-\frac{4 h h^{\prime}}{\left(h^{\prime}-h\right)^{2}}\right],
\end{aligned}
$$

where ${ }_{2} F_{1}(\alpha, \beta ; \gamma ; z)$ is the hypergeometric function [70],

$$
{ }_{2} F_{1}(\alpha, \beta ; \gamma ; z)=\sum_{j=0}^{\infty} \frac{(\alpha)_{j}(\beta)_{j} z^{j}}{(\gamma)_{j} j !} .
$$

Following the procedure outlined above, we obtain the completely general expression

$$
\begin{aligned}
\mathcal{R}_{\ell \kappa^{\prime}, \ell-1 \kappa} & \frac{(2 \ell+1) !}{2} \frac{e^{\pi \eta}\left(\kappa \kappa^{\prime}\right)^{\ell-1}}{i\left(\kappa-\kappa^{\prime}\right)^{2 \ell+2}}\left(\frac{\kappa-\kappa^{\prime}}{\kappa+\kappa^{\prime}}\right)^{1-i \eta-i \eta^{\prime}} \\
& \times\left\{\frac{\kappa+\kappa^{\prime}}{\kappa-\kappa^{\prime}}{ }_{2} F_{1}\left[\ell+1+i \eta^{\prime}, \ell+i \eta ; 2 \ell ;-\frac{4 \kappa \kappa^{\prime}}{\left(\kappa-\kappa^{\prime}\right)^{2}}\right]\right. \\
& \left.-\frac{\kappa-\kappa^{\prime}}{\kappa+\kappa^{\prime}}{ }_{2} F_{1}\left[\ell-1+i \eta^{\prime}, \ell+i \eta ; 2 \ell ;-\frac{4 \kappa \kappa^{\prime}}{\left(\kappa-\kappa^{\prime}\right)^{2}}\right]\right\},
\end{aligned}
$$

from which any bound-bound, bound-continuum, and continuum-continuum matrix elements can be obtained by the substitution $\kappa \rightarrow k$ or $\kappa \rightarrow i / n$, for continuum and bound indexes, respectively, and multiplying by the corresponding normalization factor.

\section{APPENDIX D: ASYMPTOTIC APPROXIMATION TO THE OUTER CONTINUUM-CONTINUUM TRANSITION INTEGRAL}

As discussed in Appendix A, the continuum-continuum reduced dipole matrix elements between physical states (i.e., those defined on the whole $[0, \infty)$ semiaxis) in the continuum can be computed restricting the radial integral to the interval $[0, R]$ only in the case of the acceleration gauge and provided that $R$ is sufficiently large. In the length and velocity gauge, instead, the contribution to the integral from the half-bounded interval $[R, \infty)$ cannot be neglected. If both continuum states vanish at $r=R$, one can use the expression (A17) which gives the contribution of the $[R, \infty)$ interval in terms of a boundary correction. For general energies, however, no exact expression in closed form is available, so the integral must be computed either numerically or using approximate formulas. Cormier et al. [76] derived a series expansion for such contribution. In this Appendix we examine an alternative series expansion for the outer correction to the length gauge transition matrix element, derive its analytical expression up to the third term, and analyze the performance of the formula thus obtained. Let us start from the definition

$$
\begin{aligned}
&\left.\mathcal{O}_{\ell E, \ell^{\prime} E^{\prime}}\right|_{R} ^{\infty}=\frac{C_{\ell E} C_{\ell^{\prime} E^{\prime}}}{k^{-\left(\ell+\frac{1}{2}\right)} k^{\prime-\left(\ell^{\prime}+\frac{1}{2}\right)}} \int_{R}^{\infty} d r r^{\ell+\ell^{\prime}+3} e^{i r\left(k+k^{\prime}\right)} \\
& \times{ }_{1} F_{1}\left(\ell+1-\frac{i}{k} ; 2 \ell+2 ;-2 i k r\right) \\
& \times{ }_{1} F_{1}\left(\ell^{\prime}+1-\frac{i}{k^{\prime}} ; 2 \ell^{\prime}+2 ;-2 i k^{\prime} r\right) .
\end{aligned}
$$

For $-\frac{3 \pi}{2}+\delta \leqslant \arg z \leqslant \frac{\pi}{2}-\delta$, the confluent hypergeometric functions admits the following expansion for $|z| \rightarrow \infty$ (see Eq. 13.5.1 in [70]),

$$
\begin{aligned}
& \frac{{ }_{1} F_{1}(a ; b ; z)}{\Gamma(b)} \\
& =\frac{e^{z} z^{a-b}}{\Gamma(a)}\left[\sum_{j=0}^{N-1} \frac{(b-a)_{j}(1-a)_{j}}{j ! z^{j}}+O\left(|z|^{-N}\right)\right] \\
& \quad+\frac{e^{-i \pi a} z^{-a}}{\Gamma(b-a)}\left[\sum_{j=0}^{M-1} \frac{(a)_{j}(1+a-b)_{j}}{j !(-z)^{j}}+O\left(|z|^{-M}\right)\right] .
\end{aligned}
$$

We use this expression for $M=N=3$, thus making an error $\epsilon_{F} \simeq F /(k r)^{3}$ on the evaluation of $F$, and regularize the integral (D1) introducing the parametric factor $e^{-\xi r}$. Once the integration is carried out, and after taking the limit of the result for $\xi \rightarrow 0^{+}$, we obtain an asymptotic approximation to

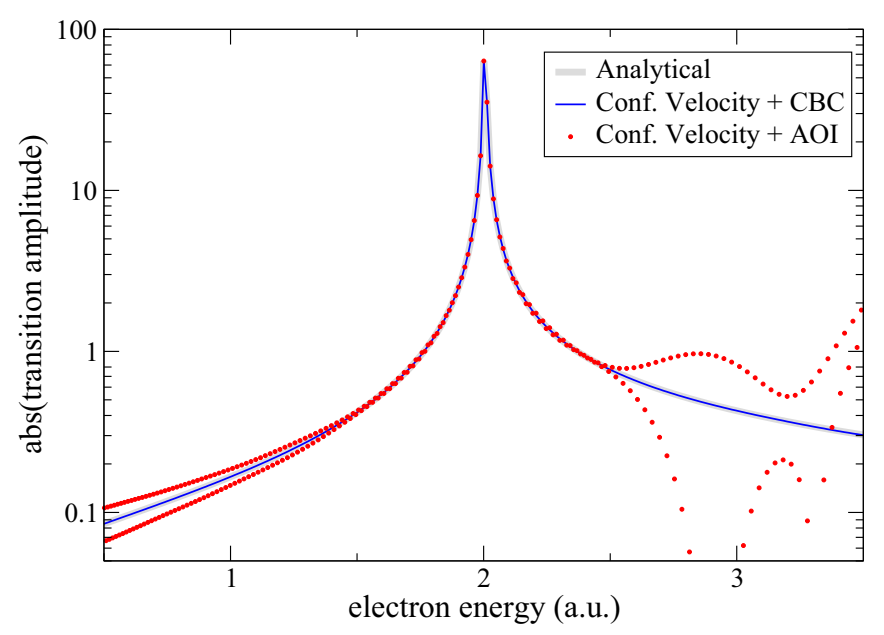

FIG. 15. (Color online) Absolute value of the reduced velocitygauge hydrogen dipole matrix element $\left\langle\psi_{E_{p}}\left\|\mathcal{O}_{1}^{(v)}\right\| \psi_{E_{s}}\right\rangle$ from the $s$ scattering state with $E_{s} \simeq 2$ a.u. to several $p$ scattering states evaluated as the sum of two contributions. The first contribution is the numerical integral shown in Fig. 6, which is computed with the GABS basis and truncated at $R_{\mathrm{box}}$, and the second contribution is either the AOI (solid circles) or the CBC (thin solid line). The thick gray solid line on the background shows the exact analytical results. See text for more details. 
the outer integral (AOI),

$$
\begin{aligned}
\left.\mathcal{O}_{\ell+1 E, \ell^{\prime} E^{\prime}}\right|_{R} ^{\infty} \approx & \frac{C_{\ell+1 E} C_{\ell E}(2 \ell+3) !(2 \ell+1) !}{(-2 i)^{2 \ell+3} \sqrt{k k^{\prime}}} \\
& \times \sum_{\sigma, \sigma^{\prime}= \pm 1} I_{\ell}\left(\sigma k, \sigma^{\prime} k^{\prime}\right),
\end{aligned}
$$

where

$$
\begin{gathered}
I_{\ell}(x, y)=2 i D_{\ell+1}(x) D_{\ell}(y)\left[G_{1}(x, y)+G_{2}(x, y)\right], \\
D_{\ell}(x)=(\operatorname{sgn} x)^{\ell+1+i / x} \frac{e^{-i\left(x R+\frac{1}{x} \ln |x| R\right)}}{x \Gamma(\ell+1-i / x)}, \\
G_{1}(x, y)=R+\frac{x+y+i x y}{x y(x+y)}\left(\frac{1}{R x y}-1\right), \\
G_{2}(x, y)=H_{1}(x, y)\left(1-\frac{1}{R x y}\right)+\frac{H_{2}(x, y)}{R}, \\
H_{1}(x, y)=h_{\ell+1}(x)+h_{\ell}(y),
\end{gathered}
$$

$$
\begin{aligned}
H_{2}(x, y)= & h_{\ell+1}(x) h_{\ell+2}(x) / 2 \\
& +h_{\ell+1}(x) h_{\ell}(y)+h_{\ell}(y) h_{\ell+1}(y) / 2, \\
h_{\ell}(x)= & (\ell+1+i / x)(\ell-i / x) /(2 i x) .
\end{aligned}
$$

Figure 15 illustrates the accuracy of the AOI (D3) by comparing the corrected reduced transition matrix element in velocity gauge for states that vanish at $R$ and $E_{s} \simeq$ 2 a.u. with the one obtained using the CBC correction given in Eq. (A17) and with the analytical result. Notice that, in this case, the correction to the length gauge can be transformed to the correction in the velocity gauge by applying the same conversion factor as in Eq. (A4). The CBC correction is very accurate as long as the truncated acceleration radial integral is converged for $r \leqslant R(R=500$ a.u. $)$. The AOI correction is extremely accurate in an energy interval of $\simeq 1$ a.u. around the diagonal, $|\Delta E|<0.5$ a.u.. The deviations between the AOI and CBC results for off-shell transitions with $|\Delta E|>$ 0.5 a.u. are attributed to the truncation of Eq. (D2) to $M=N=3$. Systematic improvement of the AOI correction is straightforward.
[1] F. Krausz and M. Y. Ivanov, Rev. Mod. Phys. 81, 163 (2009).

[2] B. W. J. McNeil and N. R. Thompson, Nat. Photon. 4, 814 (2010).

[3] A. T. J. B. Eppink and D. H. Parker, Rev. Sci. Instrum. 68, 3477 (1997).

[4] R. Dörner, V. Mergel, O. Jagutzki, L. Spielberger, J. Ullrich, R. Moshammer, and H. Schmidt-Böcking, Phys. Rep. 330, 95 (2000).

[5] Z.-H. Loh, M. Khalil, R. E. Correa, and S. R. Leone, Rev. Sci. Instrum. 79, 073101 (2008).

[6] Z.-H. Loh and S. R. Leone, J. Chem. Phys. 128, 204302 (2008).

[7] M. Dell'Angela et al., Science 339, 1302 (2013).

[8] C. Ott, A. Kaldun, P. Raith, K. Meyer, M. Laux, Y. Zhang, S. Hagstotz, T. Ding, R. Heck, and T. Pfeifer, arXiv:1205.0519.

[9] L. Argenti, C. Ott, T. Pfeifer, and F. Martín, arXiv:1211.2566.

[10] R. McWeeny, Nature (London) 166, 21 (1950).

[11] W. J. Hehre, J. Chem. Phys. 51, 2657 (1969).

[12] L. Argenti and R. Colle, Comp. Phys. Commun. 180, 1442 (2009).

[13] A. Macías, F. Martín, A. Riera, and M. Yañez, Phys. Rev. A 36, 4179 (1987).

[14] A. Macías, F. Martín, A. Riera, and M. Yañez, Int. J. Quantum Chem. 33, 279 (1988).

[15] B. M. Nestmann and S. D. Peyerimhoff, J. Phys. B: At., Mol. Opt. Phys. 23, L773 (1990).

[16] I. Cacelli, J. Phys. B: At., Mol. Opt. Phys. 30, 5643 (1997).

[17] I. Cacelli, R. Moccia, and A. Rizzo, Phys. Rev. A 57, 1895 (1998).

[18] V. Carravetta, H. Ågren, O. Vahtras, and H. J. A. Jensen, J. Chem. Phys. 113, 7790 (2000).

[19] I. Cacelli, R. Moccia, and A. Rizzo, Chem. Phys. 252, 67 (2000).

[20] A. Faure, J. D. Gorfinkiel, L. A. Morgan, and J. Tennyson, Comp. Phys. Commun. 144, 224 (2002).

[21] M. Fiori and J. Miraglia, Comp. Phys. Commun. 183, 2528 (2012).
[22] I. Cacelli, Phys. Rep. 205, 283 (1991).

[23] I. Cacelli, R. Moccia, and R. Montuoro, Phys. Rev. A 63, 012512 (2000).

[24] I. Cacelli, R. Moccia, and R. Montuoro, Chem. Phys. Lett. 347, 261 (2001).

[25] V. Feyer, P. Bolognesi, M. Coreno, K. C. Prince, L. Avaldi, B. Jansik, and V. Carravetta, J. Phys. B: At., Mol. Opt. Phys. 40, F35 (2007).

[26] R. Püttner, X.-J. Liu, H. Fukuzawa, T. Tanaka, M. Hoshino, H. Tanaka, J. Harries, Y. Tamenori, V. Carravetta, and K. Ueda, Chem. Phys. Lett. 445, 6 (2007).

[27] P. G. Burke, R-Matrix Theory of Atomic Collisions, Springer Series on Atomic, Optical, and Plasma Physics Vol. 61 (Springer, Berlin, Heidelberg, 2011).

[28] B. M. Nestmann, K. Pfingst, and S. D. Peyerimhoff, J. Phys. B: At., Mol. Opt. Phys. 27, 2297 (1994).

[29] B. K. Sarpal, K. Pfingst, B. M. Nestmann, and S. D. Peyerimhoff, J. Phys. B: At., Mol. Opt. Phys. 29, 857 (1996).

[30] T. Beyer, B. M. Nestmann, B. K. Sarpal, and S. D. Peyerimhoff, J. Phys. B: At., Mol. Opt. Phys. 30, 3431 (1997).

[31] L. A. Morgan, C. J. Gillan, J. Tennyson, and X. Chen, J. Phys. B: At., Mol. Opt. Phys. 30, 4087 (1997).

[32] L. A. Morgan, J. Tennyson, and C. J. Gillan, Comp. Phys. Commun. 114, 120 (1998).

[33] J. D. Gorfinkiel, L. A. Morgan, and J. Tennyson, J. Phys. B: At., Mol. Opt. Phys. 35, 543 (2002).

[34] I. Rozum, N. J. Mason, and J. Tennyson, New J. Phys. 5, 155 (2003).

[35] J. D. Gorfinkiel and J. Tennyson, J. Phys. B: At., Mol. Opt. Phys. 38, 1607 (2005).

[36] J. Tennyson, Phys. Rep. 491, 29 (2010).

[37] J. M. Carr, P. G. Galiatsatos, J. D. Gorfinkiel, a. G. Harvey, M. a. Lysaght, D. Madden, Z. Mašín, M. Plummer, J. Tennyson, and H. N. Varambhia, Eur. Phys. J. D 66, 58 (2012).

[38] B. I. Schneider, Phys. Rev. A 55, 3417 (1997). 
[39] J. Colgan, M. S. Pindzola, and F. Robicheaux, Phys. Rev. Lett. 98, 153001 (2007).

[40] D. A. Horner, W. Vanroose, T. N. Rescigno, F. Martín, and C. W. McCurdy, Phys. Rev. Lett. 98, 073001 (2007).

[41] L. Tao, C. W. McCurdy, and T. N. Rescigno, Phys. Rev. A 79, 012719 (2009).

[42] L. Tao, C. W. McCurdy, and T. N. Rescigno, Phys. Rev. A 80, 013402 (2009).

[43] L. Tao, C. W. McCurdy, and T. N. Rescigno, Phys. Rev. A 82, 023423 (2010).

[44] H. Bachau, E. Cormier, P. Decleva, J. E. Hansen, and F. Martín, Rep. Prog. Phys. 64, 1815 (2001).

[45] W. Vanroose, F. Martín, T. N. Rescigno, and C. W. McCurdy, Science 310, 1787 (2005).

[46] W. Vanroose, D. A. Horner, F. Martín, T. N. Rescigno, and C. W. McCurdy, Phys. Rev. A 74, 052702 (2006).

[47] J. Feist, S. Nagele, R. Pazourek, E. Persson, B. I. Schneider, L. A. Collins, and J. Burgdörfer, Phys. Rev. Lett. 103, 063002 (2009).

[48] O. a. Fojón, J. Fernández, A. Palacios, R. D. Rivarola, and F. Martín, J. Phys. B: At., Mol. Opt. Phys. 37, 3035 (2004).

[49] M. Stener and P. Decleva, J. Electron Spectrosc. Relat. Phenom. 94, 195 (1998).

[50] M. Stener and P. Decleva, J. Electron Spectrosc. Relat. Phenom. 104, 135 (1999).

[51] D. Toffoli, M. Stener, G. Fronzoni, and P. Decleva, Chem. Phys. 276, 25 (2002).

[52] D. Toffoli and P. Decleva, J. Chem. Phys. 128, 234101 (2008).

[53] S. E. Canton, E. Plesiat, J. D. Bozek, B. S. Rude, P. Decleva, and F. Martín, Proc. Natl. Acad. Sci. USA 108, 7302 (2011).

[54] L. Argenti, T. D. Thomas, E. Plésiat, X.-J. Liu, C. Miron, T. Lischke, G. Prümper, K. Sakai, T. Ouchi, R. Püttner, V. Sekushin, T. Tanaka, M. Hoshino, H. Tanaka, P. Decleva, K. Ueda, and F. Martín, New J. Phys. 14, 033012 (2012).

[55] E. Plésiat, P. Decleva, and F. Martín, J. Phys. B: At., Mol. Opt. Phys. 45, 194008 (2012).

[56] E. Plésiat, L. Argenti, E. Kukk, C. Miron, K. Ueda, P. Decleva, and F. Martín, Phys. Rev. A 85, 023409 (2012).

[57] R. K. Kushawaha, M. Patanen, R. Guillemin, L. Journel, C. Miron, M. Simon, M. N. Piancastelli, C. Skates, and P. Decleva, Proc. Natl. Acad. Sci. USA 110, 15201 (2013).

[58] D. Toffoli and P. Decleva, J. Phys. B: At., Mol. Opt. Phys. 46, 145101 (2013).

[59] S. Petretti, A. Saenz, A. Castro, and P. Decleva, Chem. Phys. 414, 45 (2013).

[60] E. Kukk, D. Ayuso, T. D. Thomas, P. Decleva, M. Patanen, L. Argenti, E. Plésiat, A. Palacios, K. Kooser, O. Travnikova, S. Mondal, M. Kimura, K. Sakai, C. Miron, F. Martín, and K. Ueda, Phys. Rev. A 88, 033412 (2013).

[61] K. Ueda, C. Miron, E. Plésiat, L. Argenti, M. Patanen, K. Kooser, D. Ayuso, S. Mondal, M. Kimura, K. Sakai, O. Travnikova, A. Palacios, P. Decleva, E. Kukk, and F. Martín, J. Chem. Phys. 139, 124306 (2013).

[62] R. Moccia and R. Montuoro, Chem. Phys. Lett. 368, 430 (2003).

[63] R. Montuoro and R. Moccia, Chem. Phys. 293, 281 (2003).

[64] T. N. Rescigno, D. A. Horner, F. L. Yip, and C. W. McCurdy, Phys. Rev. A 72, 052709 (2005).
[65] F. L. Yip, C. W. McCurdy, and T. N. Rescigno, Phys. Rev. A 78, 023405 (2008).

[66] F. L. Yip, C. W. McCurdy, and T. N. Rescigno, Phys. Rev. A 81, 063419 (2010).

[67] F. L. Yip, C. W. McCurdy, and T. N. Rescigno, Phys. Rev. A 81, 053407 (2010).

[68] C. de Boor, A Practical Guide to Splines (Applied Mathematical Sciences) (Springer, Berlin, 2001), p. 346.

[69] L. A. A. Nikolopoulos, Phys. Rev. A 73, 043408 (2006).

[70] M. Abramowitz and I. A. Stegun, Handbook of Mathematical Functions: with Formulas, Graphs, and Mathematical Tables, Dover Books on Mathematics (Dover, New York, 1965), p. 1046.

[71] R. G. Newton, Scattering Theory of Waves and Particles, 2nd ed. (Springer-Verlag, New York, 1982).

[72] A. Messiah, Quantum Mechanics. Two volumes (North-Holland, New York; Wiley \& Sons, New York, 1966), p. 1136.

[73] D. A. Varshalovich, A. N. Moskalev, and V. K. Khersonskii, Quantum Theory of Angular Momemtum (World Scientific, Singapore, 1988), p. 514.

[74] See Supplemental Material at http://link.aps.org/supplemental/ 10.1103/PhysRevA.90.012506 for extended benchmarks for Rydberg and continuum states.

[75] V. Véniard and B. Piraux, Phys. Rev. A 41, 4019 (1990).

[76] E. Cormier, H. Bachau, and J. Zhang, J. Phys. B: At., Mol. Opt. Phys. 26, 4449 (1993).

[77] S. J. van Enk, J. Zhang, and P. Lambropoulos, J. Phys. B: At., Mol. Opt. Phys. 30, L17 (1997).

[78] P. Agostini, F. Fabre, G. Mainfray, G. Petite, and N. K. Rahman, Phys. Rev. Lett. 42, 1127 (1979).

[79] E. Cormier and P. Lambropoulos, J. Phys. B: At., Mol. Opt. Phys. 30, 77 (1997).

[80] V. D. Rodríguez, D. G. Arbó, and P. a. Macri, J. Phys. B: At., Mol. Opt. Phys. 44, 125603 (2011).

[81] A. N. Grum-Grzhimailo, B. Abeln, K. Bartschat, D. Weflen, and T. Urness, Phys. Rev. A 81, 043408 (2010).

[82] L. B. Madsen, Phys. Rev. A 65, 053417 (2002).

[83] E. Cormier and P. Lambropoulos, Eur. Phys. J. D 2, 15 (1998).

[84] J. N. Bardsley, A. Szoke, and M. J. Comella, J. Phys. B: At., Mol. Opt. Phys. 21, 3899 (1988).

[85] J. Zhang and P. Lambropoulos, J. Nonlinear Opt. Phys. Mat. 04, 633 (1995).

[86] D. A. Telnov and S.-I. Chu, J. Phys. B: At., Mol. Opt. Phys. 28, 2407 (1995).

[87] E. Cormier and P. Lambropoulos, J. Phys. B: At., Mol. Opt. Phys. 29, 1667 (1996).

[88] P. V. Demekhin and L. S. Cederbaum, Phys. Rev. Lett. 108, 253001 (2012).

[89] F. H. Faisal, Theory of Multiphoton Processes (Physics of Atoms and Molecules) (Springer, Berlin, 1987), p. 408.

[90] T. Mercouris, Y. Komninos, S. Dionissopoulou, and C. A. Nicolaides, Phys. Rev. A 50, 4109 (1994).

[91] T. Mercouris, Y. Komninos, S. Dionissopoulou, and C. A. Nicolaides, J. Phys. B: At., Mol. Opt. Phys. 29, L13 (1996).

[92] Y. Komninos, T. Mercouris, and C. A. Nicolaides, Phys. Rev. A 86, 023420 (2012).

[93] B. Bransden and C. Joachain, Physics of Atoms and Molecules, 2nd ed. (Addison-Wesley, Boston, 2003).

[94] L. D. Landau and L. M. Lifshitz, Quantum Mechanics Nonrelativistic Theory, 3rd ed. (Butterworth-Heinemann, Oxford, 1976), Vol. 3, p. 689. 